\title{
Muon diffusion and trapping in aluminum and dilute aluminum alloys: Experiments and comparison with small-polaron theory
}

\author{
K. W. Kehr, D. Richter, and J. -M. Welter \\ Institut für Festkörperforschung, Kernforschungsanlage Jülich, \\ D-5170 Jülich, Federal Republic of Germany \\ O. Hartmann, E. Karlsson, and L. O. Norlin \\ Institute of Physics, University of Uppsala, S-75121 Uppsala, Sweden \\ T. O. Niinikoski \\ Conseil Européen pour la Recherche Nucléaire, CH-1211 Geneva, Switzerland \\ A. Yaouanc \\ Centre d'Etudes Nucléaires de Grenoble, Grenoble, F-38042 Grenoble, France
}

(Received 9 December 1981)

\begin{abstract}
In order to examine whether the small-polaron theory can correctly describe the motional behavior of light interstitials in metals at low temperatures, we have systematically investigated the diffusion of positive muons in Al and in Al doped with substitutional impurities of various concentrations in the temperature range between $30 \mathrm{mK}$ and 100 K. In pure $\mathrm{Al}$ ( $<1 \mathrm{ppm}$ overall impurity content) the muon was found to be mobile down to $30 \mathrm{mK}$. For doped $\mathrm{Al}$, the diffusional behavior exhibits two regimes: (i) Above $1 \mathrm{~K}$ maxima in the depolarization rate evolve, indicating a static muon in the peak regions. The position of the peaks (between 15 and $50 \mathrm{~K}$ ) is characteristic for each kind of impurity while the height and width depend on the impurity concentration. In $A / \mathrm{Mn}_{x}$ the muon occupies a tetrahedral site in this regime. (ii) Below $1 \mathrm{~K}$ the depolarization increases again with decreasing temperature and increasing impurity concentration. The temperature dependence exhibits a universal behavior independent of impurity concentration and the kind of impurity. Here the muon site is octahedral. Both regimes are interpreted in terms of diffusion-limited trapping at the impurities: (i) Above $1 \mathrm{~K}$ the diffusion is phonon assisted and entails capture rates which increase with concentration and temperature. The peak positions are determined by the escape processes from the traps which occur at characteristic temperatures for each impurity. A quantitative analysis with a two-state model for diffusion in the presence of traps revealed a linear concentration dependence of the trapping rate. A linear temperature dependence for the diffusion coefficient was found which is in strong disagreement with the $T^{7}$ prediction of conventional small-polaron theory. This linear behavior is characteristic for a one-phonon process, which should be effective only if alternating transitions between octahedral and tetrahedral sites occur. (ii) Below $1 \mathrm{~K}$, the apparent trapping rate increases with decreasing temperature, implying a faster diffusion mechanism at low temperatures. The most appealing interpretation is a trapping, preceded by a fast coherent diffusion, which is limited by muon-electron scattering. Again, in contrast to conventional small-polaron theory, which predicts $D_{\text {coh }} \propto T^{-9}$, we find proportionality to $T^{-0.6}$ for this rate.
\end{abstract}

\section{INTRODUCTION}

The diffusion of positive muons in metals has been studied intensively since the first pioneering work by Gurevich et al. ${ }^{1}$ in 1972 . It has been reviewed at two topical conferences held in 1978 (Ref. 2) and 1980 (Ref. 3) but several important problems still remain unresolved, in particular those concerning diffusion in the presence of trapping centers and diffusion at very low temperatures. One reason for the continuous interest in these phenomena is that the muon diffusion rates have turned out to be extremely sensitive to various imperfections in the metals. Another is that one 
can expect specific diffusion mechanisms, based on tunneling, if the material is sufficiently clean and the thermal vibrations are strongly reduced. At somewhat higher temperatures the muon diffusion should be similar to that of hydrogen isotopes (except for isotope effects), which evokes hopes of using the muon as a hydrogen substitute for studying diffusion, localization, and electronic structure in the very dilute limit.

Aluminum was one of the first metals that was subject to muon diffusion studies, since it is a fcc metal with large nuclear dipole moments. In fact, the depolarization rate (or linewidth) $\sigma$ arising from the dipole broadening should be very similar to that of copper, where Gurevich et al. found $\sigma=0.25 \mu \mathrm{s}^{-1}$, and a motional narrowing setting in above $100 \mathrm{~K}^{1}$ It was soon found, however, that pure aluminum gives rise to practically no depolarization of positive muons in the whole temperature range $1-300 \mathrm{~K} .^{4}$

This work deals with positive muon diffusion and localization in the purest available aluminum and in aluminum doped with small amounts (usually 5-100 ppm) of specifically selected impurities: $\mathrm{Mn}, \mathrm{Mg}, \mathrm{Li}, \mathrm{Ag}$. There have been indications ${ }^{5,6}$ that these isolated impurities will trap muons that are implanted at random in a sample, if the temperature is sufficiently high $(10-50 \mathrm{~K})$ for diffusion towards such impurity centers. These phenomena are now studied systematically for the first time by variation of the impurity content of wellcharacterized Al samples. The aim is to find out which diffusion mechanism is dominating in the $0.1-10 \mathrm{~K}$ range.

It was shown in an earlier paper by our group ${ }^{7}$ that a strong motional narrowing of the muonspin-rotation ( $\mu \mathrm{SR}$ ) signal occurs in the purest Al samples even if the temperature is as low as 0.03 $K$. This work has now been extended by including more accurate and systematic data from the same set of $A l \mathrm{Mn}_{x}$ alloys ( $\left.x=5,10,42,57,70,1300 \mathrm{ppm}\right)$ as mentioned above, one alloy with $\mathrm{Mg}(x=42 \mathrm{ppm})$, one with $\mathrm{Li}(x=75 \mathrm{ppm})$, and one with $\mathrm{Ag}$ $(x=117 \mathrm{ppm})$. These impurities were selected because they are expected to provide widely different long-range effects in the host lattice; $\mathrm{Mg}$ and $\mathrm{Mn}$ impurities producing large volume changes per atom, and $\mathrm{Li}$ or $\mathrm{Ag}$ giving nearly no such effects. The improved quality and the systematic nature of the new data should provide a basis for a discussion of those diffusion mechanisms that remain possible at very low temperatures; in particular, the question of the existence of coherent diffusion.
The diffusion data have been complemented by lattice site determinations for muons, both at the lowest temperatures $(\sim 50 \mathrm{mK})$ and in the trapping peak (at $15 \mathrm{~K}$ ) for $A l \mathrm{Mn}_{x}$.

Section II discusses first the formation of smallpolaron states of the muon and then reviews the current theories of motion of a muon: coherent and incoherent diffusion in ideal and nonideal crystals and trapping. We have felt it necessary to make this review fairly extensive in order to define the problems and to see to what extent they can be put to experimental tests. In Sec. III the experimental procedures are described, and the results of our investigations on the diffusion of muons presented. Section IV contains the experiments on the site determinations. The results are further discussed and compared with theory in Sec. V. The conclusions are summarized in Sec. VI.

\section{THEORY OF MUON MOTION}

\section{A. Small-polaron formation}

In most theoretical discussions of motion of muons in metals the muon is assumed to self-trap immediately after thermalization. Recently, however, questions on the polaron formation and a possible delay to it have been raised. Therefore, in this section we shall briefly discuss the theoretical approaches to the self-trapping process.

If a positively charged muon is implanted in a solid, thermalization occurs on a very short time scale. $^{8}$ In this process the muon will be screened by the conduction electrons of the metal, similar to the case of the proton. There remains a strong residual interaction with the host atoms mediated by the screening cloud. This interaction is often described in terms of lattice theory, where the short-range interaction is modeled by so-called Kanzaki forces $\vec{\psi}^{m}$ acting on the surrounding host atoms designated by the indices $m$. The strength of the elastic interaction between the muon and the host lattice can be parametrized in terms of the double-force tensor $P_{\mu \nu}$ which is the first moment of the Kanzaki forces,

$$
P_{\mu \nu}=-\sum_{m} \psi_{\mu}^{m} R_{v}^{m}
$$

where $R_{v}^{m}$ is the $v$ th component of a vector from the muon to the host atom $m$. In a cubic system the trace of $\overleftrightarrow{\mathbf{P}}$ is related to the volume expansion created by the lattice relaxation around a selftrapped muon by 


$$
\Delta V=\frac{\operatorname{Tr}(\stackrel{\leftrightarrow}{\mathbf{P}})}{c_{11}+2 c_{12}},
$$

where $c_{11}$ and $c_{12}$ are elastic constants of the host metal. The elastic interaction favors self-trapping and small-polaron formation. For an isotropic elastic continuum the energy gain as a result of the lattice relaxation around the interstitial muon amounts to

$$
\Delta U=-\frac{1}{2 \Omega} \frac{P^{2}}{c_{11}},
$$

where $P$ is the diagonal element of $\stackrel{\leftrightarrow}{\mathrm{P}}$ and $\Omega$ the volume of the primitive unit cell. With $P=1.42$ $\mathrm{eV}$ (cf. Sec. II C) a value of $\Delta U=-86 \mathrm{meV}$ is estimated for Al. Localization, however, also increases the kinetic energy of the muon, since higher Fourier components are involved in a localized state compared to a long-wavelength plane wave.

For the case of the positron the balance between kinetic energy and potential energy has been investigated quantitatively in the framework of continuum theory as well as of harmonic lattice theory. Both approaches lead to a kinetic-energy contribution up to 1 order of magnitude too large to allow self-trapping. On the other hand, since the kinetic energy varies as $m^{-1}$, while the elastic energy depends only weakly on $m$, for the muon as well as for the proton the ground state should be the small-polaron state.

The next problem we have to address is the question of how the muon, which initially starts in a non-self-trapped, delocalized state, does reach its equilibrium state. For the adiabatic limit, where the light particle always immediately adjusts itself to the strain pattern of the lattice, Emin ${ }^{9,10}$ investigated the existence of a barrier to self-trapping using scaling arguments. Following Emin's work, one can write the energy of a particle added to an elastic continuum as a function of a scaled position variable with scaling factor $R$. Three components contribute: (i) the kinetic energy, which scales as $T_{e} / R^{2}$, (ii) the elastic potential energy of the particle, which behaves as $-V_{\text {int }} / R^{3}$, and finally (iii) the resulting strain energy of the deformed continuum, which is given by $V_{\text {int }} / 2 R^{3}$. Thus, the total energy of the particle, which scales as

$$
E(R)=T_{e} / R^{2}-V_{\mathrm{int}} / 2 R^{3},
$$

always exhibits a maximum for finite $R$. Both an infinitely spread-out muon as well as the small polaron, which in a discrete system shrinks to typical interatomic distances, appear to be possible and are separated by a barrier. Similar results have also been obtained by Browne and Stoneham ${ }^{11}$ using a slightly different argumentation. In the context of scaling it can be easily seen that impurities which interact elastically with the muon (interaction potential $-V_{\mathrm{int}}^{\mathrm{imp}} / R^{3}$ ), reduce the barrier height.

In order to see whether the barrier causes any delay to self-trapping, the dynamic stability of the metastable non-self-trapped (free) state has to be investigated. Qualitatively it is clear that the ratio of the muon transfer time in the free state characterized by the corresponding tunneling matrix element $J_{f}$ and of the reaction time of the lattice measured by the vibrational frequencies $\omega_{D}$ of the host lattice will be the important parameter that describes the degree of adiabaticity $\boldsymbol{A}$. For small $A=z J_{f} / \hbar \omega_{D}$ (where $z$ is the coordination number of the muon), the lattice can react, and the muon will be self-trapped within a vibrational period $\sim 10^{-12} \mathrm{~s}$, while for large $A$ self-trapping will become increasingly difficult. This simple qualitative picture is supported by detailed variational studies of an electron in a deformable medium beyond the adiabatic limit. ${ }^{9}$ In addition to the role of the adiabaticity parameter these calculations show that for a given $A$ the free and the polaron states can only coexist within a certain range of $|\Delta U| / \hbar \omega_{D}$. In order to judge the significance of a possible delay to self-trapping for the muon, the rigid-lattice bandwidth has to be estimated. Taking the muon as a free particle, in analogy to a nearly free electron in a periodic lattice, a bandwidth in $\mathrm{Al}$ of about $30 \mathrm{meV}$ can be determined, which is of the same magnitude as the typical lattice frequencies. Thus $A$ is about unity and we are confronted with a borderline situation, where the theory cannot predict whether or not any delay to self-trapping will occur.

With respect to the actual experiments, the influence of temperature on the self-trapping mechanism is of great interest. Here it is useful to follow the model given by Browne and Stoneham ${ }^{11}$ of a critical fluctuation $q_{c}$ of the host atoms above which localization occurs. For a single oscillator the probability that its vibrational coordinate $q$ exceed a certain value $q_{c}$ is given by

$$
P_{T}\left(q>q_{c}\right)=\operatorname{erfc}\left(q_{c} / \widetilde{q}\right),
$$

where

$$
\widetilde{q}=\frac{\hbar}{2 M \omega} \operatorname{coth}\left[\frac{\hbar \omega}{2 k_{B} T}\right] .
$$

Expressing $q_{c}$ by the bandwidth $z J_{f}$ and the cou- 
pling energy $\Delta U$, one finds

$P_{T}\left(q>q_{c}\right)=\operatorname{erfc}\left(\frac{z J_{f}}{\left[\Delta U \hbar \omega \operatorname{coth}\left(\hbar \omega / k_{B} T\right)\right]^{1 / 2}}\right)$.

Equation (6) shows again that a delay to selftrapping can only be expected for bandwidths of the order of, or larger than, typical lattice frequencies $\hbar \omega_{D}$. In addition, an influence of temperature can only be expected for thermal energies $k_{B} T$ of the order of $\hbar \omega_{D}$. This basic result does not change if one considers the strains arising from the distribution of thermal phonons in a crystal. For example for Al metal Browne and Stoneham estimated a $14 \%$ increase of the thermal strain energy between $T=0$ and room temperature. Hence, thermal fluctuations are unlikely to provide temperature-dependent formation mechanisms at low temperatures.

We summarize the theoretical results concerning polaron formation.

(i) It is generally agreed that self-trapping of the positron does not occur.

(ii) Using the derivations leading to (i) as a basis, it follows that for heavier interstitials like the $\mu^{+}$ or the proton the polaronic state is the state of lowest energy.

(iii) While protons do always occupy this state, in the case of the muon its lifetime of $2.2 \mu \mathrm{s}$ may be less than a possible delay to self-trapping.

(iv) Self-trapping is enhanced by phonon fluctuations and by impurities or lattice defects which tend to preform self-trapping distortions. The temperature influence is expected to be weak for $T$ smaller than the Debye temperature $\Theta_{D}$.

We conclude with a remark concerning the interstitial sites occupied by muons after a smallpolaron state has formed. Some theoretical calculations for muons in Al (Refs. 12-14) suggest preference of the octahedral $(O)$ sites, compared to the tetrahedral $(T)$ sites. These results, however, are very sensitive to the pseudopotential used. Channeling experiments ${ }^{15-17}$ have indicated the $T$ sites as equilibrium positions of $\mathrm{D}$ in $\mathrm{Al}$, but this result might be due to radiation damage. In our previous study ${ }^{5}$ we have found the $T$ site in $A l \mathrm{Mn}_{0.0013}$ at $15 \mathrm{~K}$.

\section{B. Tunneling and coherent diffusion}

\section{Tunneling of small polarons}

We assume that the muon has formed a small polaron in an ideal metal crystal, i.e., it is sur- rounded by a locally relaxed lattice. This small polaron can still tunnel to a neighboring interstitial site which is related to the first site by a lattice translation. The tunneling matrix element $J_{\text {eff }}$ of this process comprises the transfer element $J$ of the muon in the rigid lattice, and the transfer of the relaxed lattice configuration between both sites. In the strong-coupling situation ${ }^{18}|\Delta U| \gg J$ which applies to $\mathrm{Al}$, the effective tunneling matrix element is obtained in the form

$$
J_{\text {eff }}=J \exp [-S(T)] .
$$

$S(T)$ is determined by the Kanzaki forces $\psi_{\mu}^{m}$ and the lattice force constants in the linear-coupling approximation and for harmonic lattices. For simple estimates valid for isotropic solids the Debye approximation of $S(0)$ is sufficient ${ }^{19}$ :

$$
S(0)=\frac{5 E_{a}}{2 \hbar \omega_{D}} .
$$

The quantity $E_{a}$ is the activation energy for incoherent hopping of small polarons (see Sec. II C), and $\omega_{D}$ is the Debye frequency. As will be detailed in Sec. II C, an indirect experimental determination gives $E_{a}=32 \mathrm{meV}$ and $J \approx 1 \mathrm{meV}$ for $\mathrm{Al}$, whereas a theoretical estimate for $E_{a}$ is $40 \mathrm{meV}$. With the value $\hbar \omega_{D} / k_{B}=428 \mathrm{~K}$ for $\mathrm{Al}$ one obtains $S(0)=1.73$ using the experimental $E_{a}$, or $S(0)=2.16$ using the estimated $E_{a}$. Hence $J$ is reduced at $T=0$ by a factor of 0.18 or 0.12 , respectively, and is estimated to be of the order of $0.1 \mathrm{meV}$ for $\mathrm{Al}$. The temperature correction of $S(T)$ behaves as $\left(k_{B} T / \hbar \omega_{D}\right)^{4}$ for small $T$, hence it is of no interest at small temperatures.

The possibility of tunneling transfer allows quantum-mechanical delocalization of a proton or muon such that in an ideal crystal at $T=0$ the proton, or a long-lived muon, would be found in an extended state of Bloch type. The width of the corresponding "small-polaron band" would be roughly given by $z J_{\text {eff }}$, where $z$ is the coordination number of the lattice ( $z=12$ for the $O$ sites of the muon sublattice in the fcc lattice). However, this small-polaron band is rather hypothetical for muons, since there are always defects in a real crystal that provide isolated localized states of low energy, i.e., that act as trapping centers. Ideally, there is at most one muon in the sample at a given time, which would always be trapped if it would not decay. The best one can hope for with respect to extended states is the formation of fairly extended wave packets of muons, which often do not reach a trap during the muon lifetime in crystals 
with very small defect concentration. It is then necessary to discuss whether the formation of extended wave packets is possible at all in a crystal with defects, far from the isolated traps. This can be done by appealing to the Anderson picture of localization.

According to Anderson ${ }^{20}$ no band states exist in a nonideal crystal when the average deviation $\Delta E$ of the ground-state energies of the interstitial sites exceeds a critical value $E_{c}$. For small-polaron bands $E_{c} \propto z J_{\text {eff }}$; the exact proportionality factor is not well known for three-dimensional lattices. ${ }^{21}$

We estimate the deviations $\Delta E$ created by point defects in the regions far from the defects from elasticity theory. We assume isotropic point defects in an anisotropic medium and use a formula given by Leibfried and Breuer, ${ }^{22}$ which represents an expansion in terms of the anisotropy

$$
\begin{aligned}
E_{\mathrm{int}}= & -\frac{1}{r^{3}} \frac{15}{8 \pi} \delta\left(\frac{\bar{c}_{11}+2 \bar{c}_{12}}{3 \bar{c}_{11}}\right)^{2} \Delta V^{r \mu} \Delta V^{d} \\
& \times\left[\frac{3}{5}-\sum_{j} \rho_{j}^{4}\right] .
\end{aligned}
$$

$\delta=c_{11}-c_{12}-c_{44}$ is the anisotropy of the medium, $\bar{c}_{12}=c_{12}+\frac{1}{5} \delta$ and $\bar{c}_{11}=\bar{c}_{12}+2 c_{44}+\frac{2}{5} \delta$ are averaged elastic constants, $\Delta V^{\mu}$ and $\Delta V^{d}$ are the volume dilatations produced by the muon and the defect, respectively, and $\rho_{j}$ are direction cosines with respect to the cubic axis. For the estimate we omit the term containing $\rho_{j}$. We use $\Delta V^{\mu}=2.9$ $\AA^{3}$, which has been found for hydrogen in fcc metals, ${ }^{23}$ and the elastic data of Al to obtain

$$
\frac{d E_{\mathrm{int}}}{d r} \approx 0.23 \frac{\Delta V^{d}}{r^{4}},
$$

in units of $\mathrm{eV} \AA^{-1}$. An estimate for the smallest value of $\Delta E$ is obtained by choosing $r$ midway between the defects and multiplying with the distance $d$ between interstitital sites. We use $\Delta V^{d}=7.4 \AA^{3}$ for $\mathrm{Mn}$; this value can be deduced from the measured lattice dilatation of $\mathrm{Mn}$ in $\mathrm{Al} .^{24}$ We obtain from Eq. (10) $\Delta E \approx 1.6 \mu \mathrm{eV}$ for $50 \mathrm{ppm}$ $\mathrm{Mn}$ and $\Delta E \approx 0.12 \mathrm{meV}$ for $1300 \mathrm{ppm} \mathrm{Mn}$. The other impurities investigated produce similar $(\mathrm{Mg})$ or considerably smaller $(\mathrm{Li}, \mathrm{Ag}$ ) lattice dilatations. The estimated lowest values for $\Delta E$ in the regions far from the defects in our samples are orders of magnitude smaller than the expected bandwidth of roughly $1 \mathrm{meV}$, except for Al with $1300 \mathrm{ppm} \mathrm{Mn}$. Hence the transient formation of extended muon states appears possible at zero temperature in all the Al samples of our study, except in the one doped with 1300 ppm Mn.

In addition to the possible destruction of extended states by static disorder, a dynamical destruction is also possible by thermal vibrations. This mechanism is closely related to the transport in extended states to which we turn our attention now.

\section{Coherent diffusion}

We consider a muon which is self-trapped at a particular interstitial site. In the band picture and at zero temperature the wave packet would spread out over the crystal. At finite temperature, a finite mean free path $l$ of the wave packet results due to scattering processes even in ideal crystals. The resulting diffusion coefficient can be represented, up to a numerical factor, as ${ }^{18}$

$$
D \cong v^{2} \tau,
$$

where $\tau$ is an inverse (transport) scattering rate and $v$ the velocity of the particle. While for electrons in metals $v$ equals the Fermi velocity, for thermalized muons one expects $v$ to be given by

$$
v \approx\left\{\begin{array}{l}
\left(3 k_{B} T / m^{*}\right)^{1 / 2}, \quad z J_{\mathrm{eff}} \gg k_{B} T \\
d J_{\mathrm{eff}} / \hbar, \quad z J_{\mathrm{eff}}<<k_{B} T
\end{array}\right.
$$

where $d$ is the distance between two interstitial sites and $m^{*}$ the effective mass of the muon. With $J_{\text {eff }} \approx 0.1 \mathrm{meV}$, one expects the first equation (12a) to hold for muons in $\mathrm{Al}$ well below $10 \mathrm{~K}$. The mean free path of a wave packet is given by $l=v \tau$; this is also a measure for the coherence length of the wave packet. The region of transport in extended states which is limited by scattering events, is commonly called "coherent diffusion."

Starting from the Liouville-von Neumann equation, Kagan and Klinger ${ }^{25}$ have derived a diffusion coefficent of the form of Eq. (11) with $v \propto J_{\text {eff }}$ corresponding to the case of Eq. (12b). They assumed from the outset that $J_{\text {eff }}$ is the smallest parameter of the problem, and that the diffusing particle is effectively localized (see below). They derived $\tau$ from an indirect phononscattering process where the localized interstitial particle is in a virtual excited state between absorption and emission of the phonon, and found proportionality with $T^{-9}$. One of the present authors ${ }^{26}$ has calculated $\tau$ from the direct phononscattering process on the localized interstitial particle. Also, a $T^{-9}$ behavior was found, and the prefactor was expressed in terms of the change of the elastic constants by adding interstitials. The rela- 
tion $\tau \propto T^{-9}$ implies $D \propto T^{-9}$ for $z J_{\text {eff }} \ll k_{B} T$ and $D \propto T^{-8}$ for $z J_{\text {eff }} \gg k_{B} T$. If the dominating phonon interaction is that with the fluctuating tunneling transfer, $D$ becomes proportional to $T^{-7}$ or $T^{-6}$, respectively.

An interaction process neglected so far (see, however, Andreev and Lifshitz ${ }^{27}$ ) is provided by the scattering of electrons on the muons. This scattering rate should be proportional to the number of thermally excited electrons, i.e., proportional to $T / T_{F}$. Furthermore, an electron can at most only transfer its thermal energy $k_{B} T$ to the muon. Consideration of energy and momentum conservation yields an additional factor $T / T_{F}$ in the scattering rate. ${ }^{28}$ Electron scattering on muons should be the dominant scattering process at low temperatures in pure crystals. Estimates of the electron-muon cross section on the basis of the Thomas-Fermi approximation lead to a scattering rate $\tau_{e}^{-1}$ of the order of $4 \times 10^{8} T^{2} \mathrm{~s}^{-1}$ when $T$ is given in $K$. The resulting diffusion coefficient should be proportional to $T^{-2}$ or to $T^{-1}$, depending on whether $z J_{\text {eff }}$ is smaller or larger than $k_{B} T$. Our estimate differs in one $T$ power from the suggestion in Ref. 27.

Scattering of wave packets on isolated defects is also possible. This process can only be dominant if other scattering processes are small, i.e., when the mean free path in the absence of scattering centers is sufficiently large. One then expects a scattering rate $\tau_{d}^{-1}=v \sigma_{\text {scatt }} c$, where $\sigma_{\text {scatt }}$ is the scattering cross section and $c$ the concentration of defects. For large mean free paths, the effect of the large de Broglie wavelength $\lambda$ of the muon at low temperatures must also be taken into account. One estimates $\lambda \approx 25 T^{-1 / 2} \AA$ ( $T$ in $\mathrm{K}$ ) for a muon with an effective mass equal to the mass of the proton. For large $\lambda$ the scattering cross section will be proportional to $v^{-1}$, and the scattering rate will be independent of temperature even in the case $z J_{\text {eff }} \gg k_{B} T$, cf. Eq. (12a).

If $\tau$ decreases with increasing temperature one readily reaches the temperatures where the mean free path $l$ is as short as the de Broglie wavelength $\lambda$ of the muon at that temperature, or as short as the distance $d$ between interstitial sites. The former case is relevant when Eq. (12a) applies, the latter when Eq. (12b) holds. According to Ioffe and Regel $^{29}$ the model of transport in extended states is no longer applicable when $l<\max (\hbar, d)$. Especially when $l$ becomes equal to $d$, the particle must be considered as localized. Kagan and Klinger maintain that in a temperature region above that point, as long as $J_{\text {eff }}$ is small, and thus the incoherent hopping rate negligible, the diffusion coefficient is still given by Eqs. (11) and (12b).

\section{Incoherent hopping of small polarons}

The coherent diffusion studied in Sec. II B 2 generally decreases with increasing temperatures, hence it will become small for larger $T$. At the same time, when $l$ becomes of the order of $d$, the muon is essentially localized at interstitial sites. A motion of a localized muon from one site to a neighboring interstitial site can now be induced by absorption (and emission) of thermal phonons. This leads to thermally assisted processes, called incoherent hopping.

\section{Hopping in ideal crystals}

Small-polaron theory ${ }^{18,30}$ predicts the following transition rate $\Gamma$ between two neighboring, equivalent interstitial sites:

(i) At low temperatures when $k_{B} T<<\hbar \omega_{D}, \Gamma$ is proportional to $T^{7}$, cf. Ref. 30. One finds for an isotropic solid

$$
\Gamma=\frac{20 \xi(4)}{\pi^{3}} \frac{P^{4} d^{4}}{\rho^{2} \hbar^{11} c_{0}^{14}} J_{\mathrm{eff}}^{2}\left(k_{B} T\right)^{7},
$$

where $\rho$ is the mass density of the host metal and $c_{0}$ the velocity of longitudinal sound. The factors in front of $J_{\text {eff }}^{2}\left(k_{B} T\right)^{7}$ can also be expressed in terms of $E_{a}$ and the Debye temperature $\Theta_{D}$ in analogy with Eq. (14). The temperature dependence is due to a two-phonon process, since in the ideal crystal a small polaron cannot absorb or emit a single phonon because of energy and momentum conservation. However, it can virtually absorb a phonon, tunnel to the neighboring site, and then emit the phonon, with the rate given by Eq. (13). Fujii $^{31}$ has obtained a $T^{3}$ behavior of the twophonon contribution to $\Gamma$ in the case of several inequivalent interstitial sites in the unit cell. has

(ii) At higher temperatures when $k_{B} T \lesssim \hbar \omega_{D}$ one

$$
\Gamma=\left(\frac{\pi}{4 E_{a} k_{B} T}\right)^{1 / 2} \frac{J^{2}}{\hbar} \exp \left(-\frac{E_{a}}{k_{B} T}\right) .
$$

A multiphonon process is necessary in order to bring the ground-state level of the occupied interstitial site to the same energy as the adjacent level. This requires the activation energy $E_{a}$. The particle can then tunnel with the transfer matrix ele- 
ment $J$; the golden rule yields the factor $J^{2}$ in Eq. (14). The remaining factor can be understood from the detailed dynamics of the coincidence of both levels. ${ }^{18}$

It is difficult to estimate the parameters $J$ and $E_{a}$ with any accuracy from the existing pseudopotential calculations for Al. An estimation of $J$ would require a precise knowledge of the potential-energy curve of the proton or muon along its diffusion path. A derivation of $E_{a}$ requires at least the knowledge of the Kanzaki forces between the proton or muon and the host atoms, and the force constants between the host atoms. Teichler ${ }^{32}$ has calculated the small-polaron transition rate between $O$ sites in a fcc lattice in a model with one spring constant $f$, and the force $g$ between a proton or muon and the nearest host atoms. He found

$$
E_{a}=0.258 g^{2} / f .
$$

Unfortunately hydrogen is not soluble in $\mathrm{Al}$, but $g$ can be estimated for protons from the empirical volume expansion by hydrogen loading, ${ }^{23}$ giving approximately $g a=1.42 \mathrm{eV}$, where $a$ is the lattice constant. In the case of $\mathrm{Cu}$, one obtains $\mathrm{g} a=2.46$ $\mathrm{eV}$, which compares well with the value of $2.64 \mathrm{eV}$ deduced by Teichler ${ }^{32}$ from diffusion data of muons in $\mathrm{Cu}$. Hence we will adopt the value given above for the further estimates. Also, the lattice expansion inferred from the values of the static linewidth (cf. Sec. IV) shows that $\Delta V$ produced by a muon is of the same order in $\mathrm{Cu}$ (Ref. 33) and $A l \mathrm{Mn} .{ }^{5}$ The spring constant $f$ for $\mathrm{Al}$ determined from $C_{44}$ is approximately $1.277 \times 10^{4} \mathrm{erg} \mathrm{cm}^{-2}$. One obtains a value of $E_{a}=40 \mathrm{meV}$ for muons from Eq. (15). This is an estimate for the activation energy for muon diffusion provided that the small-polaron hopping process is effective between octahedral sites in the fcc lattice.

So far, thermally activated hopping of muons in pure Al has not been observed directly as a change of the depolarization rate with $T$; it seems to be too rapid in the temperature range of interest. Hence a direct experimental determination of $J$ by application of small-polaron theory has not been possible, contrary to the case of $\mathrm{Cu}$. An indirect estimate of $J$ has become possible by the observation of muon trapping in vacancies in Al by Herlach $^{34}$ at higher temperatures. Assuming diffusion-limited trapping, he could verify the validity of Eq. (14) with $E_{a}=32 \mathrm{meV}$, which is in fair agreement with the estimate given above. With the use of Eq. (19) for the rate of diffusionlimited trapping we have estimated the prefactor of
Eq. (14) from his data and found $J$ to be of the order of $1 \mathrm{meV}$. This is a fairly large value compared to the one for muons in $\mathrm{Cu}$, where $J \approx 18$ $\mu \mathrm{eV}$ has been obtained. ${ }^{32}$

\section{Hopping in nonideal crystals}

In a nonideal crystal with defects, the exact lattice translation invariance has been destroyed and the sites are no longer equivalent. Apart from the fact that defects provide trapping centers (to be studied in Sec. II E) there are energy differences $\Delta E$ between neighboring sites even far from the defects. These differences have been estimated in Sec. II B. Here one-phonon processes can contribute to the transport since such energy differences allow absorption or emission of single phonons. The transition rate between two sites with energy differences $\Delta E$ and transfer matrix element $J$ was first derived by Sussmann. ${ }^{35}$ Some modifications are necessary in order to include small-polaron effects; the corresponding transition rate has been deduced by Teichler and Seeger, ${ }^{36} \mathrm{cf}$. also Ref. 37 . Their results contain contributions from even and odd combinations of the double force tensors of the muon in its initial and final state. For diffusion between equivalent sites only the even combination contributes and after applying an isotropic Debye approximation, their Eq. (9) yields for phonon absorption $(\Delta E>0)$

$$
\Gamma_{\mathrm{abs}}=\frac{J_{\mathrm{eff}}^{2} P^{2}(\Delta E)^{2} d^{2}}{12 \pi \rho \hbar^{6} c_{0}^{7}} \frac{\Delta E}{\exp \left(\Delta E / k_{B} T\right)-1} .
$$

For phonon emission, $\Delta E$ must be replaced by $|\Delta E|$, and the rate must be multiplied by $\exp \left(|\Delta E| / k_{B} T\right)$. For $k_{B} T \gg|\Delta E|$ one has

$$
\Gamma_{\mathrm{abs}} \approx \Gamma_{\mathrm{em}} \approx \frac{J_{\mathrm{eff}}^{2} P^{2}(\Delta E)^{2} d^{2}}{12 \pi \rho \hbar^{6} c_{0}^{7}} k_{B} T,
$$

i.e., a rate proportional to $T$. The rate Eq. (17) depends explicitly on the magnitude of the energy differences, contrary to the result found for reorientation of defects. ${ }^{35}$

If the crystal is sufficiently pure, the contribution from Eqs. (16) and (17) will die out, and onephonon processes are only possible if odd combinations of the double force tensors $\overleftrightarrow{\mathbf{P}}_{i}$ and $\overleftrightarrow{\mathbf{P}}_{j}$ in the initial and final site contribute to the rate. They are nonzero if the double-force tensors are not the same in both sites. This can happen if the interstitial particle is diffusing between sites of different symmetry $(T \rightarrow O \rightarrow T \rightarrow O \rightarrow \cdots)$ or if it is not 
located in the centers of the interstitial sites. The latter case appears to be very unlikely since up to now $\mathrm{H}$ has always been found to be in a symmetry position in undisturbed metals. Starting from Teichler and Seeger ${ }^{36}$ and applying again an isotropic Debye approximation we obtain the onephonon transition rate for this case in a high- $T$ approximation $\left(\Delta \epsilon<<k_{B} T\right.$, where $\Delta \epsilon$ is now the energy difference between the two different interstitial sites):

$$
\Gamma=\frac{J_{\text {eff }}^{2}\left[\operatorname{Tr}\left(\overleftrightarrow{\mathrm{P}}_{j}\right)-\operatorname{Tr}\left(\stackrel{\leftrightarrow}{\mathrm{P}}_{i}\right)\right]^{2}}{2 \pi \rho \hbar^{4} c_{0}^{5}} k_{B} T,
$$

plus a term which is equivalent to Eq. (17).

Although Eq. (18) does not contain $\Delta \epsilon$ explicitly it is a consequence of an energy difference between the sites considered. If equivalent sites of an ideal crystal are made inequivalent by the introduction of defects, the difference of the traces in Eq. (18) would also depend on the strength of the disturbances.

\section{Transition between coherent and incoherent diffusion; summary}

Assuming the theoretical expressions given above for coherent and incoherent diffusion, one can predict the transition temperature $T_{t}$ below which coherent diffusion prevails and above which incoherent hopping dominates. If the same phononinteraction process would delimit coherent diffusion at lower temperatures and promote hopping at higher temperatures, $T_{t}$ would coincide with the temperature above which extended states are no longer meaningful. The pertinent process is the interaction of the phonons with the effective tunneling transfer. Holstein ${ }^{18}$ has discussed in this way the temperature-dependent lifetime of band states and the concomitant transition to incoherent hopping. Also, Petzinger ${ }^{38}$ has developed a unified theory of coherent and incoherent diffusion; his predictions of $T_{t}$ are some tenths of the Debye temperature of the host crystal. Additional interaction processes tend to further reduce coherent diffusion without promoting incoherent processes at higher temperatures, hence the actual transition temperature should be lower than estimated from the argument above. For example, Kagan and Klinger $^{25}$ have considered intrawell scattering of phonons on a localized interstitial, but they did not give an explicit prediction of $T_{t}^{\mathrm{ph}}$. The rate derived from the direct phonon-scattering process leads to an estimate of $T_{t}^{\mathrm{ph}}=34 \mathrm{~K}$ in $\mathrm{Cu}$ (Ref. 26) and similar values are expected for the other fcc metals.

In summary, the main predictions of the theory of small-polaron motion in crystals are as follows.

(i) Diffusion occurs at low temperature through coherent transfer processes, resulting in a diffusion coefficient which in general increases with decreasing temperature.

(ii) At higher temperatures diffusion takes place via thermally activated hopping of localized small polarons. The explicit behavior with temperature depends on the process which is most effective in delimiting coherent transfer or promoting hopping. We have collected the various predictions for temperature dependencies in Table I. We emphasize that the ranges of validity are not included in the table; the main text should be consulted for their consideration.

\section{E. Capture and release at trapping centers}

It has been demonstrated by various experiments that defects can act as trapping centers for muons, ${ }^{39-41}$ hence the motion of muons is strongly influenced by them. We will describe transport of muons in the presence of traps by a two-state model. In this model a particle spends an average time $\tau_{1}$ in the state of diffusion; it is then captured by a trap and released from the trap after a mean time $\tau_{0}$. The implications of these processes on muon depolarization will be discussed in Sec. III E. In this section we will discuss in more detail the parameters $\tau_{0}, \tau_{1}$ of the two-state model. It is also possible to generalize the two-state model to an $n$ state model, in order to take several kinds of traps into account.

The escape out of a trap is only ${ }^{42}$ possible by thermal activation, hence the escape rate $\tau_{0}^{-1}$ must exhibit this feature. The activation energy should be characteristic for the detailed structure of the trapping center, i.e. specific for the kind of defect or impurity introduced. The escape rate $\tau_{0}^{-1}$ should be independent of the concentration of the trapping centers, except for secondary influences at higher concentrations. For structured traps the final escape results from several steps, and no simple relation between the total activation energy and that required for the individual steps is expected.

The capture rate $\tau_{1}^{-1}$ is sensitive to the nature of the transport processes which lead a muon towards a trapping center. In the case of hopping of localized muons, the theory of diffusion-controlled trapping $^{43}$ can be applied, with the result 
TABLE I. Temperature dependence of scattering rates and diffusion coefficients for small-polaron motion.

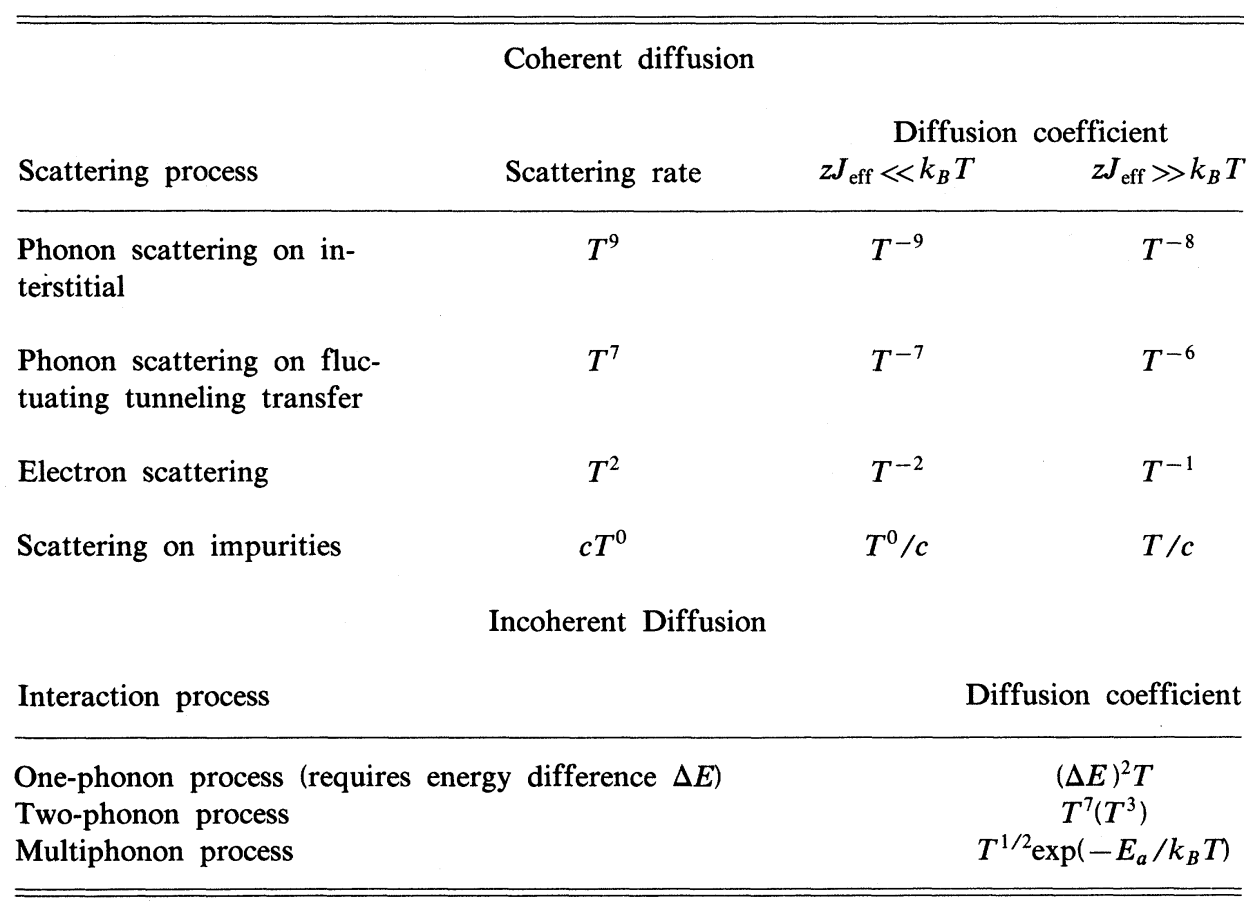

$$
\tau_{1}^{-1}=4 \pi r_{t} D n_{t} .
$$

Here $r_{t}$ is the trapping radius and $n_{t}$ the number of trapping centers per unit volume. Equation (19) is valid for a random walk with mean free path $l \ll r_{t}$. In the case of random walk on a regular lattice with randomly distributed traps a similar result holds. In the case of extended traps, $r_{t}$ is an effective radius determined by the condition that the lowering of the saddle-point energies is approximately equal to $k_{B} T$, leading to ${ }^{44} r_{t} \propto T^{-1 / 3}$.

In the case of coherent transport, when $l \gg r_{t}$, the capture rate is given by ${ }^{45}$

$$
\tau_{1}^{-1}=\sigma v n_{t}
$$

where $v$ is the mean velocity of the muon and $\sigma$ the absorption cross section. For sufficiently long mean free path, the large de Broglie wavelength $\lambda$ of the muon at temperatures below $1 \mathrm{~K}$ must also be taken into account (cf. Sec. II B 2). For large $\lambda$ and deep traps the absorption cross section $\sigma$ is proportional to $v^{-1}$; thus $\tau_{1}^{-1}$ becomes independent of $v .^{45}$ The result is a temperature-independent capture rate, as has been found for positrons trapped by vacancies. ${ }^{46}$ Positrons have a much smaller mass than the muons, but on the other hand the temperature of our investigations is much lower than in a typical positron experiment, hence similar conditions with respect to $\lambda$ should hold. For more shallow traps, the effective absorption cross section can depend on temperature in a complicated way. The behavior of the capture rate in the intermediate region where $l \approx r_{t}$ is not well understood; one expects a smooth interpolation between Eqs. (19) and (20).

\section{EXPERIMENTS ON MUON DIFFUSION}

\section{A. Preparation of samples}

Because the dynamics of muons depends very sensitively on chemical and structural imperfections, high-quality samples are needed to obtain reliable experimental results. In the following the techniques used to prepare and to characterize such samples for the present investigations will be described.

Aluminum with less than $1 \mathrm{ppm}$ of metallic impurities was used as base material (brand Kryal R- 
$\mathrm{OZ}$ from Vereinigte Aluminium Werke AG, Bonn). The residual resistivity ratio of this material varied between 15000 and 30000 . The nominal purities of the alloying elements were as follows: $\mathrm{Ag}, 99.9999 \%$; $\mathrm{Li}^{7}, 99.85 \% ; \mathrm{Mg}, 99.998 \%$; and $\mathrm{Mn}, 99.99 \%$. The alloys were prepared by highfrequency (HF) induction melting. To obtain homogeneous samples in the 100 -at. ppm range an intermediate master alloy was used. The crucibles were made from high-purity alumina. Before melting the casting unit was evacuated to $10^{-5}$ Torr, flushed with argon, reevacuated, and pressurized with 1400 Torr of an $\mathrm{Ar}-4 \mathrm{vol} . \% \mathrm{H}_{2}$ gas mixture. The melt was cast into graphite-coated stainless-steel molds to form blocks with the dimensions $15 \times 7 \times 2 \mathrm{~cm}^{3}$. From these blocks the polycrystalline samples (dimensions $3 \times 3 \times 2 \mathrm{~cm}^{3}$ ) or the blanks for the single crystals were machined. Single crystals with a diameter of 1.3 or $1.5 \mathrm{~cm}$ were grown by the Bridgman technique. The material of the crucible was either graphite or boron nitride. [110]-oriented seeds were connected to the blanks by electron-beam welding. Crystals with a length of approximately $10 \mathrm{~cm}$ were grown at a rate of $0.5 \mathrm{~cm} / \mathrm{h}$. The atmosphere in the growth unit consisted of high-purity argon at a pressure of 1200 Torr which was introduced into the apparatus after various pump-down cycles to $10^{-5}$ Torr. The crystals were usually sectioned into three pieces by spark cutting. After rechecking the orientation of each individual piece with $\mathrm{x}$-rays, they were mounted to form the specimens.

Three techniques were employed to characterize the samples. The structural quality was assessed by diffractometry with $412-\mathrm{keV} \gamma$ rays. At every centimeter along the crystal axis the rocking curve due to a slice with a thickness of $0.1 \mathrm{~cm}$ was measured. Usually widths at half-height below $30^{\prime \prime}$ were obtained. In only a few cases they reached values of $120^{\prime \prime}$. The concentration of the alloying element was determined by atomic absorption spectroscopy. From each individual piece of material up to three probes were taken and dissolved in a $\mathrm{HNO}_{3}-\mathrm{HCl}$ mixture. At least three independent concentration determinations were made for each solution. The results are listed in Table II. The level of residual impurities was checked by sparksource mass spectrometry. Test samples were made by cutting a pair of pins from the bulk and by turning them on a lathe with a diamond tool. No etchant was used. Each pair of pins was analyzed four times. The average results for some samples are reported in Table III. The symbol < means well below. The reported values are considered to be on the high-level side. For the doping elements $\mathrm{Ag}, \mathrm{Li}, \mathrm{Mg}$, and $\mathrm{Mn}$ the results obtained by atomic absorption spectrometry are much more reliable. Because the mass spectrometer was adjusted for the high masses, a quantitative analysis of the $\mathrm{Li}$ line was not performed. The high carbon levels probably result from the use of the diamond tool. No figure is given for oxygen because the interference with the surface oxide was strong and erratic. The level of dissolved oxygen is estimated to be below $1 \mathrm{ppm}$. The high magnesium level may be correlated with the high vapor pressure of this element. The iron level in the $A l \mathrm{Mn}$ sample is probably an artifact due to the re-

TABLE II. Determination of the concentration of the alloying elements in aluminum by atomic absorption spectroscopy.

\begin{tabular}{|c|c|c|c|c|}
\hline $\begin{array}{l}\text { Alloy } \\
\text { designation }\end{array}$ & $\begin{array}{l}\text { Nominal } \\
\text { concentration } \\
\text { (at.ppm) }\end{array}$ & $\begin{array}{l}\text { Measured } \\
\text { concentration } \\
\text { (at. ppm) }\end{array}$ & $\begin{array}{c}\text { Number of } \\
\text { sample } \\
\text { uptakes }\end{array}$ & $\begin{array}{c}\text { Number of } \\
\text { determinations }\end{array}$ \\
\hline AlAg & 117 & $116.8 \pm 3.8$ & 2 & 6 \\
\hline$A l \mathrm{Li}$ & 75 & $75.8 \pm 4.0$ & 2 & 10 \\
\hline$A / \mathrm{Mg}$ & 42 & $41.5 \pm 4.0$ & 1 & 3 \\
\hline$A l \mathrm{Mn}$ & 5 & $4.6 \pm 0.8$ & 1 & 7 \\
\hline$A l \mathrm{Mn}$ & 10 & $9.9 \pm 0.1$ & 1 & 6 \\
\hline$A l \mathrm{Mn}$ & 42 & $41.8 \pm 5.6$ & 1 & 10 \\
\hline$A l \mathrm{Mn}$ & 57 & $57.6 \pm 2.0$ & 3 & 20 \\
\hline$A l \mathrm{Mn}$ & 70 & $69.9 \pm 1.6$ & 2 & 6 \\
\hline$A l \mathrm{Mn}$ & 1300 & $1137.0 \pm 53.0$ & 3 & 12 \\
\hline$A l \mathrm{Mn}$ & 1300 & $1296.0 \pm 87.0$ & 2 & 15 \\
\hline
\end{tabular}


TABLE III. Determination of impurities in high-purity aluminum and different aluminum alloys by spark-source mass spectrometry. The concentrations are given in at. ppm.

\begin{tabular}{|c|c|c|c|c|c|}
\hline & Pure Al & $A l \mathrm{Ag}$ & $A l \mathrm{Li}$ & $A l \mathrm{Mg}$ & $A l \mathrm{Mn}$ \\
\hline $\mathrm{Li}$ & $<0.02$ & $<0.3$ & & $<0.08$ & $<0.08$ \\
\hline $\mathrm{Be}$ & $<0.02$ & $<0.06$ & $<0.6$ & $<0.06$ & $<0.06$ \\
\hline B & 0.006 & $<0.1$ & $<0.5$ & $<0.4$ & $<0.5$ \\
\hline $\mathrm{C}$ & 6.0 & $<0.2$ & $3.1 \pm 1.9$ & $1.2 \pm 0.9$ & $5.8 \pm 1.8$ \\
\hline $\mathbf{N}$ & 0.32 & $0.8 \pm 0.5$ & $0.7 \pm 0.2$ & $0.1 \pm 0.02$ & $0.2 \pm 0.02$ \\
\hline $\mathrm{F}$ & 0.024 & $<0.3$ & $<0.4$ & $<0.7$ & $<0.6$ \\
\hline $\mathrm{Ne}$ & & $<0.3$ & $<0.1$ & $<0.3$ & $<0.3$ \\
\hline $\mathrm{Na}$ & 0.015 & $0.2 \pm 0.04$ & $1.0 \pm 0.3$ & $<0.5$ & $0.1 \pm 0.04$ \\
\hline $\mathrm{Mg}$ & 1.5 & $1.0 \pm 0.04$ & $0.9 \pm 0.2$ & $521 \pm 270$ & $<2$ \\
\hline $\mathrm{Si}$ & $<0.1$ & $<\overline{0.7}$ & $<0.3$ & $<1$ & $<1$ \\
\hline $\mathbf{P}$ & $<0.1$ & $<0.5$ & $<0.2$ & $<0.4$ & $0.02 \pm 0.1$ \\
\hline $\mathbf{S}$ & 0.89 & $<0.5$ & $<0.9$ & $<0.4$ & $0.05 \pm 0.2$ \\
\hline $\mathrm{Cl}$ & $<0.1$ & $<0.3$ & $<0.6$ & $<0.2$ & $<0 . \overline{2}$ \\
\hline $\mathrm{Ar}$ & & $<0.1$ & $<0.1$ & $<0.1$ & $<0.2$ \\
\hline $\mathrm{K}$ & 0.83 & $<0.5$ & $<0.3$ & $<0.1$ & $<0.6$ \\
\hline $\mathrm{Ca}$ & 0.27 & $<0.2$ & $<0.3$ & $0.1 \pm 0.01$ & $<0.5$ \\
\hline Sc & $<0.02$ & $<0.1$ & $<0.6$ & $<0.5$ & $<0.3$ \\
\hline $\mathrm{Ti}$ & $<0.3$ & $<0.3$ & $<0.6$ & & $<0.3$ \\
\hline V & $<0.15$ & $<0.3$ & $0.5 \pm 0.02$ & & $<0.2$ \\
\hline $\mathrm{Cr}$ & $<0.06$ & $<0.5$ & $<0.5$ & & $1.1 \pm 0.4$ \\
\hline $\mathrm{Mn}$ & $<0.025$ & $<0.2$ & $<0.5$ & & $115 \pm 19$ \\
\hline $\mathrm{Fe}$ & 0.2 & $0.3 \pm 0.08$ & $0.2 \pm 0.02$ & & $4.0 \pm 1.6$ \\
\hline Co & $<0.1$ & $<0.4$ & $<0.3$ & & $<0.1$ \\
\hline $\mathrm{Ni}$ & $<0.15$ & $<0.2$ & $<0.3$ & & $<0.5$ \\
\hline $\mathrm{Cu}$ & $<0.025$ & $0.2 \pm 0.07$ & $0.4 \pm 0.1$ & & $0.2 \pm 0.08$ \\
\hline $\mathrm{Zn}$ & $<0.11$ & $<\overline{0.1}$ & $0.6 \pm 0.2$ & & $<0.5$ \\
\hline $\mathrm{Ga}$ & 1.8 & $<0.5$ & $<0.5$ & & \\
\hline $\mathrm{Ag}$ & $<0.3$ & $94 \pm 33$ & & & \\
\hline
\end{tabular}

action of a $\mathrm{Mn}^{+}$ion with the hydrogen of the residual-gas atmosphere. A conservative conclusion is that the amount of all the residual impurities is less than 5 at.ppm and that the level of metallic impurities should not exceed 2 at. ppm. Finally, one should keep in mind that the major part of these elements are segregated at structural sinks.

\section{B. Experimental setup}

The experiments were performed in the muon beams at the $600-\mathrm{MeV}$ synchrocyclotron at CERN, Geneva. Two different beam lines and experimental setups for $\mu$ SR have been employed in this investigation. The CJ1 beam is the more intense, with typical count rates of $\sim 500$ events/s. This figure is reached in spite of the fact that the effective detector solid angle must be limited to $\sim 15 \%$ because of the high positron contamination. The second beam line, the LJ1 beam, can give count rates of $\sim 300$ events/s with a solid angle of $\sim 30 \%$. This beam is much cleaner, with lower background, but can occasionally give problems with bad time structure.

In these experiments the detectors were normal plastic scintillators (NE 102) put together to make one in-beam muon-sensitive telescope and two or three positron-detector telescopes. In the positroncontaminated CJ2 beam a perspex Cerenkov detector was added to eliminate positrons by means of logical anticoincidences.

Ordinary leading-edge discriminators were employed for time definition since a time resolution of $3-4 \mathrm{~ns}$ is far from critical at the maximum magnetic field applied in these experiments $(1500$ G). Time histograms were collected by direct time to digital conversion at $500 \mathrm{MHz}$ or $1 \mathrm{GHz}$ clock frequency. ${ }^{47}$ The number of events collected varied 
from $10^{6}$ to $3 \times 10^{6}$. In the more intense $\mathrm{CJ} 2$ beam a pile-up logic was always used to remove events where two muons were stopped in the sample within $10 \mu \mathrm{s}$. This reduced the rate of data collection by $\sim 30 \%$. In the $\mathrm{LJ} 1$ beam pile-up rejection had no noticeable effect and was therefore sometimes omitted.

The CJ2 beam line is equipped with an iron magnet and a ${ }^{4} \mathrm{He}$ continuous-flow cryostat, capable of reaching temperatures down to $2 \mathrm{~K}$. The lower-temperature data were taken in the $\mathrm{LJ} 1$ beam in a ${ }^{3} \mathrm{He}-{ }^{4} \mathrm{He}$ dilution refrigerator, which can cool the $\mu \mathrm{SR}$ samples to $30 \mathrm{mK}$; the maximum temperature that can be stabilized accurately in this setup is around $15 \mathrm{~K}$. Therefore some overlap of the temperature ranges is possible. The magnetic field in the LJ1 beam is given by a Helmholtz coil. At present the maximum field is limited to 1400 $\mathrm{G}$, which is enough, however, to essentially decouple the electric field gradient on the $\mathrm{Al}$ nuclei in the $A l \mathrm{Mn}$ alloys.

The temperature was measured with calibrated germanium, carbon, and platinum resistors, and was stabilized within $0.2-1.0 \%$. In the dilution refrigerator problems arose below $100 \mathrm{mK}$, since our calibrated Ge resistor, attached directly to the sample, lost heat contact below that temperature. On the other hand, carbon resistors were sensitive to beam heating in this temperature range. Reproducible results for the lowest temperatures could be best obtained by reading a carbon resistor placed on the sample holder a few centimeters out of the central beam spot. It was quite clear that the holder and sample were in good thermal contact, since the measured $\mu \mathrm{SR}$ damping parameter responds to the holder temperature changes all the way down to lowest temperatures. Also, in the absence of a beam a carbon resistor attached to the sample showed a temperature compatible to that of the holder. The low-temperature measurements on the $A l \mathrm{Mn}$ 5-ppm sample were unfortunately made with a reversed-resistor arrangement, and the derived temperatures are in this case based on comparisons with a third resistor placed far away from the sample. Above $100 \mathrm{mK}$ no such problems were found.

The orientation of the single crystals was checked with Laue backscattering after the mounting on the sample holders. The accuracy of the orientation including the mounting in the cryostats is $2^{\circ}-3^{\circ}$. In the ${ }^{4} \mathrm{He}$ cryostat the samples can be rotated around an axis perpendicular to the magnetic field and the beam direction. Thus it is possible by mounting the single crystals with the [110] direction parallel to the axis of rotation (for cubic lattices) to bring any of the main crystal directions [100], [110], or [111] to coincide with the direction of the magnetic field. In the dilution refrigerator this rotation is not possible, and the single-crystal samples had to be taken out and reoriented on the sample holder.

\section{Data treatment}

Transverse-field muon-precession data are normally analyzed with the expression

$N=N_{0} \exp \left(-t / t_{\mu}\right)\left[1+a_{0} P(t) \cos (\omega t+\phi)\right]+B g$,

where $t_{\mu}=2.20 \mu \mathrm{s}$ is the mean lifetime of the muon, $\omega$ is the precession frequency with initial amplitude $a_{0}$, and $B g$ is the accidental (flat) background. The damping of the polarization $P(t)$ is typically taken as either exponential $\exp (-\lambda t)$ or Gaussian $\exp \left(-\sigma^{2} t^{2}\right)$. In practice a more complete expression is always used at CERN:

$$
\begin{aligned}
N=N_{0} \exp \left(-t / t_{\mu}\right)\{1+ & {\left[a_{0}^{\prime} P(t)+b_{0} f_{b}(t)\right] } \\
& \times \cos (\omega t+\phi)\}+B g .
\end{aligned}
$$

Here $a_{0}^{\prime}$ is the effectively observable asymmetry from the sample and $b_{0}$ is the asymmetry originating from muons stopping in regions around the sample (sample holder, cryostat walls, etc.). This fraction is never negligible in the CERN beams; $b_{0}$ is typically between 0.02 and 0.06 depending on sample size, etc., while the total observable asymmetry $a_{0}$ ranges from 0.18 to 0.26 .

This precessing background component is characterized by an asymmetry $b_{0}$ and a damping function $f_{b}$, while the frequency can be taken as equal to that of the muons in the aluminum samples. Empirically it has been found that a Gaussian damping $f_{b}(t)=\exp \left(-\sigma_{b}^{2} t^{2}\right)$ is a good representation of the background function. The parameters $b_{0}$ and $\sigma_{b}$ were determined by replacing the actual sample by a dummy sample of stainless steel having the same dimensions and mass, in order to ensure the same absorption conditions as in the real measurements. The stainless steel becomes antiferromagnetic below $40 \mathrm{~K}$, and no precession signal from the dummy sample can then be seen. Therefore the remaining precession signal gives the background from the surroundings, under similar conditions as in the real measurements.

A complementary way to obtain information 
about the precessing background is to reduce the temperature to well below the superconducting transition temperature $(\sim 1 \mathrm{~K})$ while keeping the aluminum samples in zero magnetic field. A weak magnetic field (below $100 \mathrm{G}$ ) is then turned on, and the small precession signal from the surroundings can be observed. This procedure will however only yield $b_{0}$ since $\sigma_{b}$ is affected by the magnetic field distortion around the superconducting sample. Since, however, $\sigma_{b}$ is very well known for the LJ1 setup, this procedure could be used in a few cases in order to save the time for sample changes, which is quite substantial with the dilution refrigerator.

\section{Results of the measurements}

As mentioned in the Introduction, pure aluminum does not show any measurable depolarization down to low temperatures. The temperature range has been extended by our group down to $30 \mathrm{mK}^{7,48}$ and the depolarization rate has been found negligible down to $100 \mathrm{mK}$. The low-temperature results for the damping parameter $\sigma$ for pure $\mathrm{Al}$ have been included in Fig. 1.

The dramatic effects of doping aluminum with small amounts of impurities can be seen in Fig. 1. Here the depolarization rate $\sigma$ is displayed for various concentrations of mangenese impurities, and compared to the results from undoped aluminum. The damping parameter $\sigma$ is used, which follows from the assumption of Gaussian depolarization $P(t)=P_{0} \exp \left(-\sigma^{2} t^{2}\right)$. More details on the

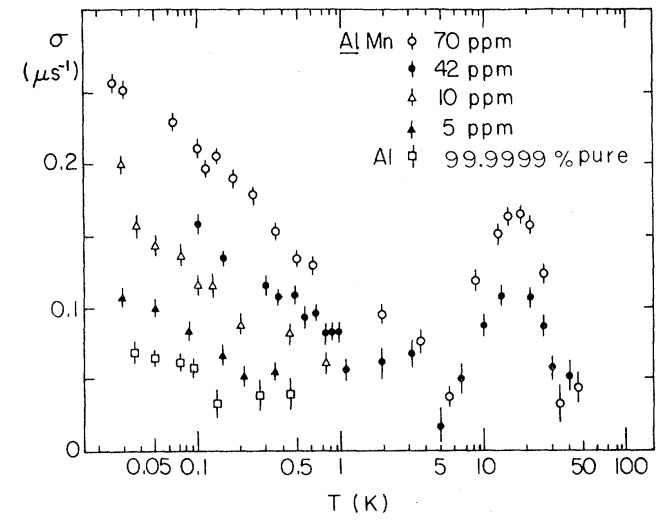

FIG. 1. Gaussian damping parameter $\sigma$ for $\mathrm{Al}$ and $A I \mathrm{Mn}$ polycrystalline samples. The external field is $B_{0}=520 \mathrm{G}$ for $42 \mathrm{ppm} A l \mathrm{Mn}, 150 \mathrm{G}$ for the others. depolarization process can be found in Sec. III E. Values of $\sigma$ below $0.05 \mu \mathrm{s}^{-1}$ can hardly be distinguished from zero depolarization within the statistics of the measurements. The small increase in $\sigma$ for the $99.9999 \%$ pure aluminum sample below $100 \mathrm{mK}$ is, however, believed to be a real effect, presumably reflecting the influence of the residual $\sim 1$ ppm impurities.

The temperature dependence appearing in Fig. 1 can for convenience be separated into two different regions-one below $1 \mathrm{~K}$ where $\sigma$ is roughly proportional to $\ln (1 / T)$, and another above $1 \mathrm{~K}$ where a characteristic peak in the depolarization rate appears. At still higher impurity concentrations the two regions are not as well separated as can be seen from Fig. 2, where the results from a 1300ppm sample are shown together with other singlecrystal results. The 1300-ppm sample was measured several times, and the data included in the figure contain several measurements with the field in the [111] direction. In fact, these data are in reality coming from two samples, one with 1137 ppm and the other with $1296 \mathrm{ppm} \mathrm{Mn}$, but since we do not find any significant difference in the $\mu \mathrm{SR}$ results we use $1300 \mathrm{ppm}$ as a common notation.

The temperature and concentration dependence of $\sigma$ in the region above $1 \mathrm{~K}$ shows the typical features of capture and release of muons from traps. Figure 3 shows this region in more detail

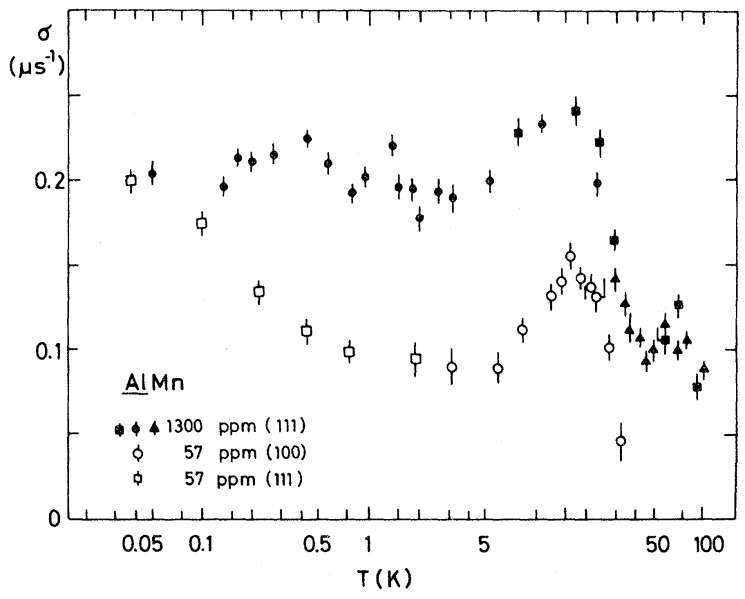

FIG. 2. Gaussian damping parameter $\sigma$ for $A l \mathrm{Mn}$ single-crystal samples. The $1300-p p m$ sample was measured in a $(220-250)-G$ field, the others in a (130-150)-G field. At these field values there should only be small differences between the different orientations (cf. Fig. 7). 
for the $A l \mathrm{Mn}$ samples of various concentrations. The 1300-ppm sample shows clear indications of saturation, i.e, around $15 \mathrm{~K}$ practically all muons remain in traps during the time of observation. In this sample a secondary peak seems to appear at higher temperatures. Similar extra peaks have been seen in other strongly doped aluminum and niobium samples. $6,39,49$

The effect of doping aluminum with other kinds of impurity atoms can be seen in Fig. 4 . In the region above $1 \mathrm{~K}$ this appears as a shift of the characteristic temperature for the trapping peak, i.e., the escape process starts around $20 \mathrm{~K}$ in $A l \mathrm{Mn}$ and $A l \mathrm{Ag}$ but around $50 \mathrm{~K}$ in $A l \mathrm{Li}$ and $A l \mathrm{Mg}$. The $A l \mathrm{Mg}$ results should be compared to a measurement by Kossler et al. on an $A l \mathrm{Mg}$ sample with higher concentration $(1000 \mathrm{ppm}){ }^{6}$ The characteristic escape temperature is the same, and even the indications of a double structure in the peak is similar for the two different concentrations.

While the trapping peak appears at temperatures seemingly characteristic for each kind of impurity,

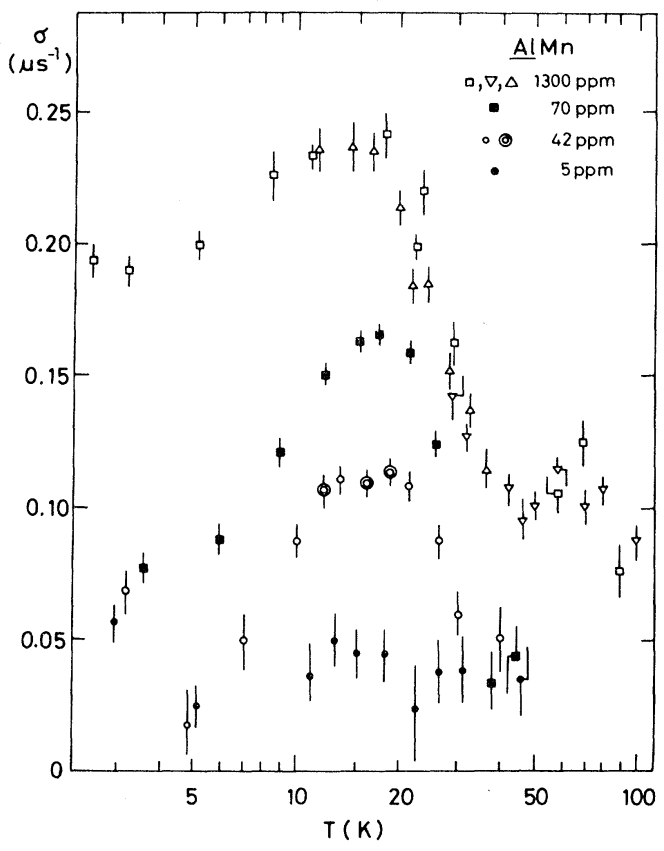

FIG. 3. Trapping peak region around $15 \mathrm{~K}$ for $A l \mathrm{Mn}$ samples of various concentrations. Some of the samples have been measured at more than one magnetic field, which is indicated by different symbols. The 42-ppm points are at higher field than the others except for the three points marked with a double ring. These were measured at $130 \mathrm{G}$ and scaled down $15 \%$ in order to accomodate for the field dependence of the linewidth.

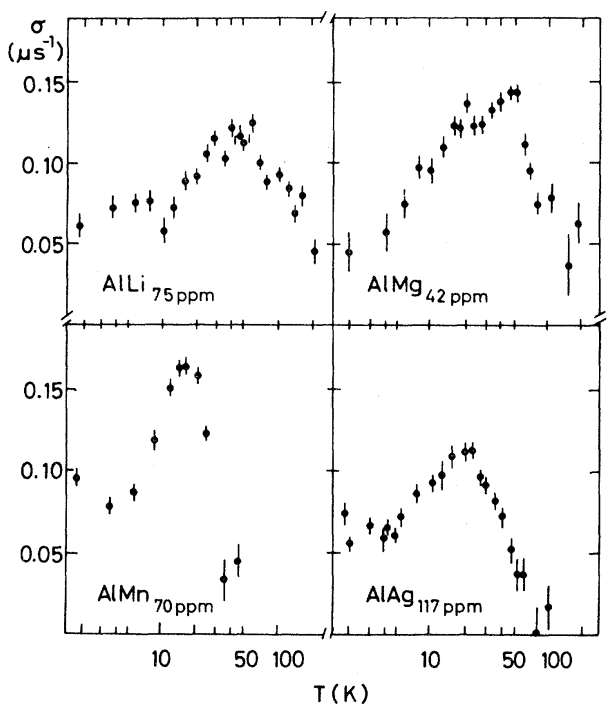

FIG. 4. Gaussian damping parameter $\sigma$ for $\mathrm{Al}$ with various doping elements for $T=2-100 \mathrm{~K}, B_{0}=150 \mathrm{G}$.

the low-temperature data below $1 \mathrm{~K}$ are very similar for $A l \mathrm{Mn}, A l \mathrm{Li}$, and $A l \mathrm{Ag}$. Figure 5 shows this part of the temperature range, and it is evident that the functional form of the temperature dependence is identical for the different impurities. The $\mathrm{Mn}$ atoms appear to produce a stronger depolarization per impurity atom than $\mathrm{Li}$ and $\mathrm{Ag}$. This may be related to the local lattice distortion around the impurity, where $\mathrm{Li}$ and $\mathrm{Ag}$ act only locally, giving practically zero net volume expansion.

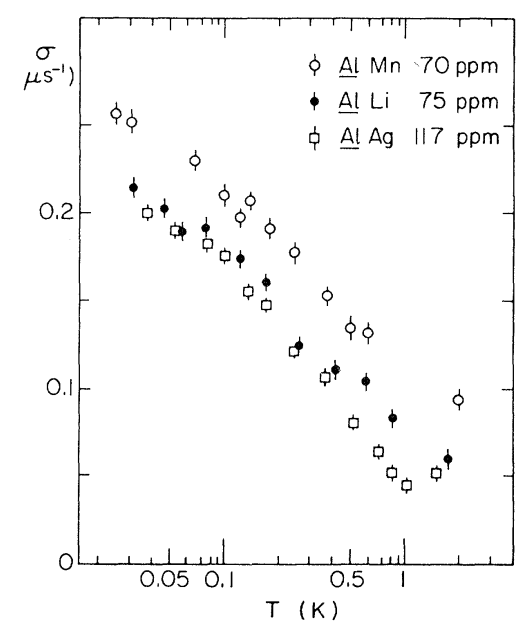

FIG. 5. Gaussian damping parameter $\sigma$ for $A l \mathrm{Mn}$, $A l \mathrm{Li}$, and $A l \mathrm{Ag}$ samples below $2 \mathrm{~K}$ (at $B_{0}=150 \mathrm{G}$ ). 


\section{E. Evaluation of results above $1 \mathrm{~K}$}

\section{Depolarization through incoherent hopping, including trapping}

As has been noted above, the measured depolarization parameter $\sigma$ in the $\mathrm{Al}$ alloys above $1 \mathrm{~K}$ shows features which are characteristic of trapping and detrapping processes. In order to analyze the data further we need a relation between the observed depolarization and the hopping processes, including trapping. Since these topics have been covered in detail in several publications ${ }^{39,50-53}$ we only indicate the main results of the theory.

An immobile, pointlike muon shows in good approximation a Gaussian depolarization (normalized to unity):

$$
P(t)=\exp \left(-\sigma^{2} t^{2}\right) .
$$

The width of the Gaussian $\sigma$ is related to the dipolar interactions of the host atoms with the muon, cf. Sec. IV A. If localized muons perform hopping motion, they are under the influence of different dipolar fields during their lifetime. The rotation frequency $\omega^{\prime}$ of the transverse muon spin due to the dipolar fields becomes time dependent. One usually assumes a Gaussian Markov process for $\omega^{\prime}(t)$ with a correlation time $\tau_{c}$, which should be proportional to the mean residence time of a muon on a particular site. The polarization decay resulting from this assumption is ${ }^{54,55}$

$$
P(t)=\exp \left\{-2 \sigma^{2} \tau_{c}^{2}\left[\exp \left(-t / \tau_{c}\right)-1+t / \tau_{c}\right]\right\} .
$$

For $\sigma \tau_{c} \gg 1$, i.e., practically immobile muons, one obtains from this equation the static result, Eq. (23), whereas for $\sigma \tau_{c} \ll 1$, corresponding to rapid motion, one finds exponential decay which represents motional narrowing,

$$
P(t)=\exp \left(-2 \sigma^{2} \tau_{c} t\right) .
$$

A "strong-collision" model where $\omega$ ' changes its values abruptly leads essentially to the same results. $^{55,51}$ One of the authors ${ }^{56}$ has numerically investigated the relation between the residence time, $\tau$, and $\tau_{c}$. It was found that $\tau$ is very close to $\tau_{c}$ for $O$ and $T$ sites in fcc lattices while they can differ considerably for bcc lattices for high applied fields.

The influence of capture and release processes on the depolarization of the muon spin in the case of incoherent hopping can be treated in the framework of the two-state model outlined in Sec. II E.
According to this model, a muon diffuses in a free state for a mean time $\tau_{1}$, and then spends a mean time $\tau_{0}$ in the trap, etc. This process is similar to the elementary processes of the strong-collision model, and that model is also easily extended to include trapping and detrapping. ${ }^{51,52}$ The depolarization decay is determined by two coupled integral equations that can be solved explicitly in the Laplace domain. The solution for $P(t)$ in the time domain is then obtained by numerical transformation. Since this procedure is somewhat unwieldy for use in fitting routines we actually used the approximate treatment of the polarization decay within the two-state model by a set of two coupled differential equations. ${ }^{39}$ As has been shown in Ref. 51 the solutions of the differential equations are always similar to the solutions of the integral equations; especially similar are the resulting damping parameters of both approaches, which differ only slightly. Other derivations of the polarization decay for diffusion in the presence of traps have also been given; e.g., Petzinger, Munjal, and Zaremba ${ }^{53}$ have developed a Gaussian-Markovian theory for $P(t)$.

\section{Results for the parameters}

In order to obtain the physical parameters for the trapping region above $1 \mathrm{~K}$ we have fitted the data with the differential equations for the twostate model of Ref. 39 . The fitting was actually done in two steps by first deriving a $\sigma$ value from the theoretical $P(t)$ function, and then comparing the experimental and theoretical $\sigma$ values in a least-squares fit. Here we have used the value $\sigma_{0}=0.265 \mu \mathrm{s}^{-1}$ for the damping in the absence of motion when fitting the data taken in $150 \mathrm{G}$ magnetic field, while the $A l \mathrm{Mn} 42-\mathrm{ppm}$ sample measured in $500 \mathrm{G}$ was fitted using $\sigma_{0}=0.22 \mu \mathrm{s}^{-1}$. In principle a simultaneous fit of several measurements with the static $\sigma$ as a free parameter could be attempted, but this has not been done with the present data.

The differential equations of Ref. 39 were solved under the assumption that the muon diffusion in the free state is rapid enough to give zero polarization. In this case $\tau_{c}$ is very small and the equations of Ref. 39 can be simplified. The resulting systems of equations were solved by a RungeKutta method and used in the least-squares fits to the experimental $\sigma$ values. The temperature dependence of the escape rate was taken as an Arrhenius-type process $\tau_{0}^{-1}=\Gamma_{0} \exp \left(-E_{a} / k_{B} T\right)$, 
while the capture rate $\tau_{1}^{-1}$ appears almost proportional to $T$. In the fits we used $\tau_{1}^{-1}=\Gamma_{1} T^{\alpha}$ with the exponent $\alpha$ as a free parameter, which was found to be slightly below 1 for the $A l \mathrm{Mn}$ samples. The data from three different concentrations were fitted simultaneously with a common temperature dependence but different prefactors. The results of the fit are found in Table IV.

The fitting of the trapping peaks in the case of the other impurities is complicated by the fine structure visible in the linewidth as function of temperature, and the parameters summarized in Table IV are to some extent an average description of the situation. A fit with several trapping centers as in Refs. 6 and 49 was not considered worthwhile.

\section{F. Evaluation of results below $1 \mathrm{~K}$}

\section{Depolarization through coherent motion}

As mentioned in Sec. II there are reasons to believe that coherent processes may be effective below $1 \mathrm{~K}$. We must therefore consider the interpretation of the depolarization in the region where coherent diffusion prevails. Although the theory of depolarization has only been partially worked out for this case, we try to outline the behavior of $\sigma(T)$ for coherent diffusion, followed by trapping in impure crystals.

The polarization $P(t)$ of the spin of the muons can be expressed in terms of the time-dependent correlation function of the magnetic fields acting on the muon. ${ }^{55,57}$ Assuming that there is no reaction of the muons on the dipolar fields and that $T_{1}$ processes can be neglected, McMullen and Zarem$\mathrm{ba}^{57}$ showed that the decay rate of $P(t)$ is determined by the "self-diffusion function" $\left\langle\hat{n}(\overrightarrow{\mathrm{r}}, t) \hat{n}\left(\overrightarrow{\mathrm{r}}^{\prime} t^{\prime}\right)\right\rangle$ where $\hat{n}(\overrightarrow{\mathrm{r}}, t)$ is the density opera- tor of a muon at site $\overrightarrow{\mathrm{r}}$ at time $t$. For sufficiently long times the decay rate is governed by the correlation time $\tau_{c}$ defined by

$$
\tau_{c}=\int_{0}^{\infty} d\left(t-t^{\prime}\right)\left\langle\hat{n}(\overrightarrow{\mathrm{r}} t) \hat{n}\left(\overrightarrow{\mathrm{r}} t^{\prime}\right)\right\rangle .
$$

For incoherent hopping $\tau_{c}$ is proportional to the mean residence time on a site. For coherent diffusion in the transition region to incoherent jumps, where the extension of the wave packets is still small, $\tau_{c}$ should be given by the effective time of transfer to neighboring site, i.e.,

$$
\tau_{c} \approx a^{2} / D_{\mathrm{coh}}
$$

If $\tau_{c} \sigma \ll 1$, the polarization decay in this region should correspond to motional narrowing according to Eq. (25) with $\tau_{c}$ given by Eq. (27). The derivations of McMullen and Zaremba suggest the validity of Eq. (27) also for the case of large mean free path of the muons.

The purest aluminum shows virtually complete absence of depolarization down to $100 \mathrm{mK}$; in other words, there is practically no structure in $\sigma(T)$ which could be compared with theory. In order to deal with the impure samples, depolarization in the case of coherent diffusion in the presence of trapping centers must be considered explicitly. This can be done again in the framework of the twostate model. Here two simplifications can be made:

(i) Complete motional narrowing can be assumed during the coherent diffusion of the muon in the undisturbed regions of the crystal, in view of the findings for pure Al.

(ii) Release processes from the traps can be neglected at the temperatures considered, since they would require thermal activation.

Then, the main source of depolarization is the trapping of the muons which is determined by the trapping rate $1 / \tau_{1}$. Contrary to depolarization

TABLE IV. Fitting parameters of the two-state model for the different Al samples.

\begin{tabular}{lccccr}
\hline \hline Sample & $\begin{array}{c}\text { Conc. } \\
(\mathrm{ppm})\end{array}$ & $\Gamma_{1}\left(\mathrm{~s}^{-1}\right)$ & $\alpha$ & $\Gamma_{0}\left(\mathrm{~s}^{-1}\right)$ & $E_{a}(\mathrm{~K})$ \\
\hline$A l \mathrm{Mn}$ & 42 & $1.62(12) \times 10^{4}$ & & & \\
$A l \mathrm{Mn}$ & 57 & $2.02(14) \times 10^{4}$ & $0.89(3)$ & $2.86(35) \times 10^{7}$ & $120(6)$ \\
$A l \mathrm{Mn}$ & 70 & $2.43(14) \times 10^{4}$ & & & $0.85(15) \times 10^{6}$ \\
$A l \mathrm{Li}$ & 75 & $4.0(10) \times 10^{4}$ & $0.36(7)$ & $2.03(44) \times 10^{6}$ & $145(27)$ \\
$A l \mathrm{Mg}$ & 42 & $3.3(4) \times 10^{4}$ & $0.54(4)$ & $2.45(32) \times 10^{6}$ & $142(7)$ \\
$A l \mathrm{Ag}$ & 117 & $3.9(5) \times 10^{4}$ & $0.45(5)$ & $87(7)$ \\
\hline \hline
\end{tabular}


during the diffusion process, where the damping is proportional to $\tau_{c}$ [see Eq. (25)], it is now roughly proportional to $1 / \tau_{1}$. Considering Eq. (19) this means that here the damping is governed by $1 / \tau_{c}$.

\section{Results}

We have first fitted the experimental data below $1 \mathrm{~K}$ by the formula (24), which interpolates between Gaussian depolarization and motional narrowing but assumes a uniform type of motion over the time of observation. Figure 6 shows the inverse correlation times $\tau_{c}^{-1}$ for the different $A l \mathrm{Mn}$ samples. In this double-log plot the results from an individual fit of the 5-, 10-, 42-, and 70-ppm samples are presented as solid and dashed lines. The best common fit to the data is

$$
\tau_{c}^{-1}=6.9(1.2) T^{0.60(4)} c^{-0.76(4)} \times 10^{5} \mathrm{~s}^{-1},
$$

where $T$ is given in $\mathrm{mK}$ and $c$ in ppm. The possible interpretations of such a straightforward application of Eq. (24) are discussed in Sec. V.

As suggested above, we have also fitted the data by the two-state model. The differential equations of Ref. 39 were used and fast diffusion in the free state $\left(\tau_{c}^{-1} \rightarrow \infty\right)$ together with negligible escape

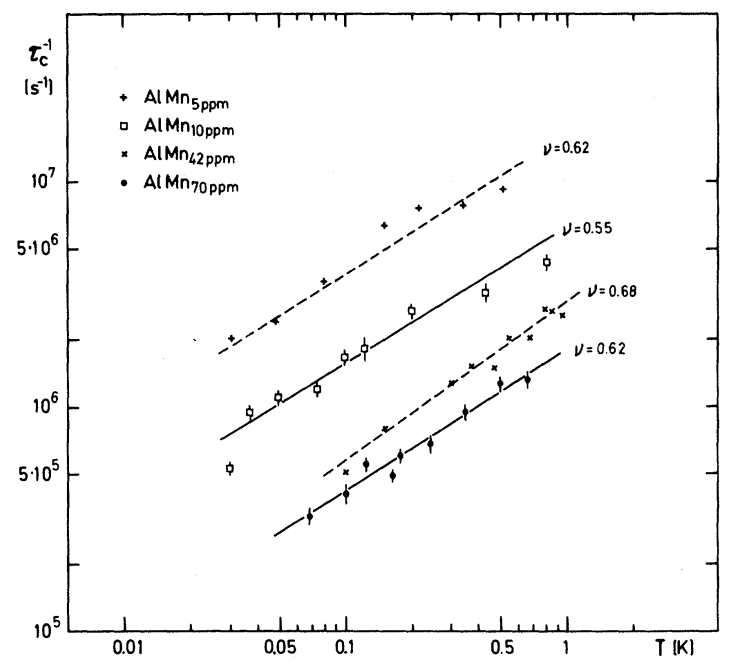

FIG. 6. Inverse correlation times $\tau_{c}^{-1}$ for various $A l \mathrm{Mn}$ samples as function of temperature. The $\tau_{c}$ values were evaluated from Eq. (24). The lines are individual fits to a $T^{v}$ dependence with the parameter $v$ given in the figure. A global fit of the data gives $v=0.60(4)$. $\left(\tau_{0}^{-1} \rightarrow 0\right)$ were assumed. The result of the fit of $\tau_{1}^{-1}$ is then

$$
\tau_{1}^{-1}=3.0(6) T^{-0.61(4)} c^{0.76(4)} \times 10^{6} \mathrm{~s}^{-1} .
$$

Compared with Eq. (28), $\tau_{1}^{-1}$ exhibits reversed signs of the exponents of $T$ and $c$, as it is expected from Sec. III F 1.

The fits in Eqs. (28) and (29) were made directly to the full depolarization function $P(t)$. This is one of the reasons for the slightly different values of the exponential factors from the ones given in Ref. 48. The temperature scale has also been slightly corrected, especially for the 5-ppm sample, where the experimental uncertainty is large.

\section{EXPERIMENTS ON SITE DETERMINATION}

\section{A. General remarks}

$\mu \mathrm{SR}$ offers the possibility to determine the symmetry of the interstitial site where the muon is located by investigating the dependence of the linewidth $\sigma$ on magnetic field and crystal orientation. We consider immobile pointlike muons that show Gaussian depolarization according to Eq. (23). $\sigma$ is determined by the dipolar fields created by the host metal atoms at the site of the muon. Van Vleck ${ }^{58}$ first evaluated the second moment of the distribution of dipolar fields in the context of NMR. His results as applied to muons is

$$
\sigma^{2}=\frac{1}{6} \gamma_{\mu}^{2} \gamma_{H}^{2} \hbar^{2} S(S+1) \sum_{j} \frac{\left(3 \cos ^{2} \theta_{j}-1\right)^{2}}{r_{j}^{6}} .
$$

$S$ is the spin of a host atom, $\gamma_{H}$ its gyromagnetic ratio, $r_{j}$ the distance between the muon and the host atom $j$, and $\theta_{j}$ the polar angle of the vector $\vec{r}_{j}$ with respect to the $\overrightarrow{\mathbf{B}}$ axis.

For a single crystal, according to Eq. (30), $\sigma$ depends on the orientation of the crystal with respect to $\overrightarrow{\mathrm{B}}$. Values for $\sigma$ are quite different for the main symmetry directions [100], [110], and [111] in cubic metals and depend strongly on the type of interstitial site. Hence a determination of the type of the site is possible through the measurement of $\sigma$, by orienting the crystal in different symmetry directions. Hartmann ${ }^{59}$ pointed out that this consideration only holds for large magnetic fields, since at small fields contributions of the electric field gradient created by the muon itself can come into play. As a charged impurity, the muon 
creates an electric field gradient (EFG) at the neighboring host nuclei. When these nuclei have a quadrupole moment, a new quantization axis of the nuclear moments has to be determined from the combined magnetic and electric interactions. The result for the dipolar width $\sigma$ depends strongly on the relative interaction $b=\omega_{0} / \omega_{E}$, where $\omega_{E}$ is an electric interaction frequency determined by the quadrupole moment and the strength of the EFG. For small $b$ the EFG interaction dominates and $\sigma$ is nearly isotropic; for $b \gg 1$ the van Vleck values [Eq. (30)] are obtained. Aluminum possesses a quadrupole moment $Q=0.15 \mathrm{~b}$, and the ratio of the quadrupole to magnetic moment is somewhat lower than in copper. It should therefore be quite easy to quench the electric interaction by applying magnetic fields. The crossover from electric to magnetic field behavior occurs around $b=5$; for a detailed discussion see Ref. 59. The field dependence of the depolarization rate $\sigma$ can also be used to estimate the strength of the electric field gradient produced on the surrounding lattice nuclei.

\section{B. Experimental results}

The first measurement of the field dependence in aluminum was made on the 1300 -ppm singlecrystal sample. Here the puzzling fact emerged that at $15 \mathrm{~K}$ the muon site appears to be tetrahedral, while at lower temperatures $(2-7 \mathrm{~K})$ there seems to be a mixture of octahedral and tetrahedral interstitial sites. ${ }^{5}$ Since then we have made several other measurements on single crystals, and the present situation is illustrated in Fig. 7. The data indicate at the lowest temperatures a practically pure octahedral site, while the intermediate region is a mixture, and finally the trapping peak at $15 \mathrm{~K}$ has tetrahedral symmetry as before.

The absolute values of the $\sigma$ 's are smaller than the calculated values for pointlike muons in undistorted environments. This effect is about $30 \%$ for the 1300-ppm sample at $15 \mathrm{~K}$, where we believe that all muons are trapped and a static linewidth is observed. As in the case of copper ${ }^{33}$ this can be ascribed to either a local lattice dilatation $(\sim 10 \%)$ or a local spread of the muon wave function.

The quadrupole frequency $\omega_{E} / 2 \pi$ derived from the 1300 -ppm sample at $15 \mathrm{~K}$ is $0.051 \mathrm{MHz}$. This corresponds to a field gradient of $q=0.40 \AA^{-3}$ at nearest neighbors. The previous value given in Ref. 5 was numerically wrong by a factor of 2 . The uncertainty in the determination of $\omega_{E}$ is

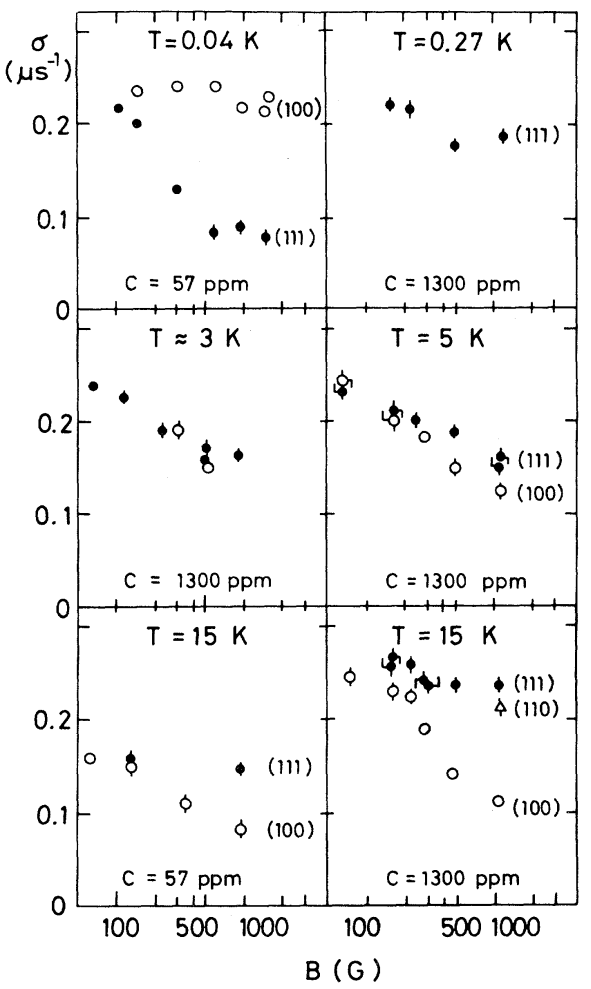

FIG. 7. Magnetic field dependence of the damping parameter $\sigma$ for several $A l \mathrm{Mn}$ samples and temperatures. The 0.04-K data shows an octahedral interstitial site symmetry, while the two measurements from $15 \mathrm{~K}$ show a tetrahedral site symmetry.

around $15 \%$, and in addition, errors in the quadrupole moment will affect the uncertainty in $q$.

The electric field gradient at the lowest temperature was also derived from the 57-ppm data (octahedral sites). The value at nearest neighbors is $0.30 \AA^{-3}$. This somewhat lower value at the octahedral position could just reflect the increased muon-aluminum distance (the $r^{-3}$ dependence assumed for $q$ leads to a relative decrease of 0.65 compared to the tetrahedral site).

In the previous discussion we have assumed that the muon is in a pure aluminum dipole environment. This is certainly a good approximation for the $A l \mathrm{Mn}$ samples, where the $\mathrm{Mn}$ atoms have about the same magnetic moment as the aluminum atoms. In addition the field gradient created by $\mathrm{Mn}$ on neighboring $\mathrm{Al}$ atoms is known to be 0.45 $\AA^{-3}$. Although the exact behavior of the muon linewidth in this complicated situation with the two different sources of field gradients has not been calculated, we have previously drawn the conclusion that the muon can hardly be a nearest 
neighbor to the $\mathrm{Mn}$ atom.

We have also studied the magnetic field dependence of the $A l \mathrm{Mg} 42-\mathrm{ppm}$ polycrystal at $40 \mathrm{~K}$. The measurement does not give as clear information as a single crystal, since the site can not be determined and the maximal change in $\sigma$ with $B$ is only $32 \%$. However, the data indicate that the electric field gradient is somewhat smaller in $A l \mathrm{Mg}$ at $40 \mathrm{~K}$ than in $A l \mathrm{Mn}$ at $15 \mathrm{~K}$. The reason for this is not clear-a different site preference is a possibility. Experimental problems may also have influenced this measurement.

There is also the possibility that an electronic moment at the $\mathrm{Mn}$ atom contributes to the linewidth of the muon. It is not certain whether $A l \mathrm{Mn}$ can be considered as a Kondo system. But if this is the case, the Kondo temperature is 700 $\mathrm{K}$, which leads to spin fluctuations of the order of $10^{14} \mathrm{~s}^{-1}$. This rapid fluctuation rate gives a negligible contribution to the depolarization (less than $0.001 \mu \mathrm{s}^{-1}$ ) even if the muon is at the nearest interstitial site to the Mn atom. Also, the similarity of the depolarization rate in Fig. 5 obtained for $A l \mathrm{Ag}$ and $A l \mathrm{Mn}$ invalidates that any paramagnetic effects are from Mn.

\section{INTERPRETATION}

As was noted in the presentation of the results the observations fall naturally into two categories, reflecting essentially different processes above and below $T=1 \mathrm{~K}$, respectively. The same division will be kept as we now attempt to interpret the data in somewhat more detail.

\section{A. Region above $1 \mathrm{~K}$}

The salient features of muon depolarization in Al crystals with impurities above $1 \mathrm{~K}$, can be summarized as follows:

(a) There are maxima in the damping rate $\sigma(T)$.

(b) The heights of the maxima increase with the concentration of the impurities.

(c) The positions of the maxima and the detailed shape of $\sigma(T)$ are specific for each kind of impurity introduced.

These observations constitute clear evidence that muons, which diffuse by thermally activated processes at these temperatures, are captured and released by the impurities. Some points of this general picture need more detailed consideration.
The data for $\mathrm{Mn}$-doped $\mathrm{Al}$ and the analysis by the two-state model show that the capture rate $\tau_{1}$ is proportional to $c$. Hence the trapping takes place at single impurities (except for $c=1300 \mathrm{ppm}$ as will be discussed below). The two-state model can also be used to extract some information on the nature of the traps. We consider Al with 70 ppm $\mathrm{Mn}$ as a particular example. At a temperature of $17 \mathrm{~K}$ corresponding to the maximum of $\sigma(T)$ the fit by the two-state model gives $\tau_{1}=3.3$ $\mu \mathrm{s}$ and $\tau_{0}=40 \mu \mathrm{s}$.

Not every muon is caught by a trap during its lifetime in this sample at $17 \mathrm{~K}$; however, when it is trapped it will rarely escape. The mean equilibrium population of traps corresponding to these values is $92 \%$. (Owing to the nonequilibrium initial condition and the finite lifetime of the muons the actual average population of the trapping sites will be reduced compared to this value.)

On the other hand, if one estimates the mean equilibrium population for trapping sites having a binding energy corresponding to $120 \mathrm{~K}$ (cf. Sec. III) from Boltzmann statistics, one finds a value of only $7.5 \%$ at $17 \mathrm{~K}$ for this sample. We ascribe this apparent discrepancy to the structure of the trapping centers. Each impurity creates a region containing several sites where muons are effectively caught. The comparison of the thermal and the two-state values of the populations suggests a factor of the order of 100 trapping sites for each $\mathrm{Mn}$ atom. Extended traps can also modify the activation energy for escape, i.e., the activation energy seen in the depolarization process can be smaller than the binding energy. Analogous effects have been observed in a neutron scattering experiment on $\mathrm{H}$ in $N b \mathrm{~N}_{x} \cdot{ }^{60}$ In the sample with $1300 \mathrm{ppm}$ $\mathrm{Mn}$ one finds almost no indication of motional narrowing below $17 \mathrm{~K}$. This can also be attributed to the existence of extended regions around the $\mathrm{Mn}$ atoms where the motion of the muons is strongly hindered.

Another experimental fact supporting the picture of extended traps is the value of the electric field gradient derived for the muon sites at $15 \mathrm{~K}$ in the $\mathrm{Mn}$-doped samples. It is characteristic for a $T$ site in a pure $\mathrm{Al}$ environment. It is therefore improbable that a $\mathrm{Mn}$ atom is a nearest neighbor.

Herlach $^{34}$ has studied the trapping of muons on vacancies in $\mathrm{Al}$ produced by electron irradiation and found no indications of trapping for 5-ppm vacancies in the temperature range under discussion, whereas for $5 \mathrm{ppm} \mathrm{Mn}$ clear indications of trapping are observed. We conclude that the trapping radius of $\mathrm{Mn}$ exceeds that of vacancies con- 
siderably.

The application of the theory of diffusionlimited trapping to the experimentally determined trapping rate leads to a diffusion constant nearly proportional to $T$, for temperatures between $1 \mathrm{~K}$ and the maximum of $\sigma(T)$. The fitted exponent of $T$ in

$$
\tau_{1}^{-1} \propto T^{\beta}
$$

is $\beta=0.89(3)$. This temperature dependence is in obvious disagreement with the theoretical predictions for incoherent hopping, cf. Sec. II C, i.e., no $T^{7}$ behavior is seen. On the other hand, it is in qualitative accordance with the rate expected from one-phonon processes. For the strain-induced part of the one-phonon process the diffusion rate is proportional to $(\Delta E)^{2}$, where $\Delta E$ is the energy difference between strain-split levels, cf. Eqs. (16) and (17). The average $(\Delta E)^{2}$ should increase with increasing impurity concentration; a rough estimate is proportional with $c^{2}$. Practically no dependence on the concentration of impurities is seen in the hopping rate deduced from the capture rate after the trivial concentration factor of Eq. (19) has been divided out. In addition, the jump rates should be very different for impurities with large and small long-range strain fields. The data for the different impurities used in this study lead to quite similar jump rates, in contradiction to the expectation (cf. Table IV).

There remains the possibility of one-phononinduced transitions between crystallographically inequivalent sites, for instance, transition sequences $O-T-O-T-O-T$ in the fcc lattice as described by Eq. (18). Such processes would be independent of strain energy differences $\Delta E$ as long as $\Delta E \ll E_{O}$ $-E_{T}=\Delta \epsilon_{O T}$, and could give rise to a linear $T$ dependence for $k_{B} T \gg \Delta \epsilon_{O T}$.

A comparison between the trapping curves for impurities with different volume expansions $(\mathbf{M n}$ and $\mathrm{Mg}$ on one hand and $\mathrm{Li}$ and $\mathrm{Ag}$ on the other) shows only a partial correlation between the long- range strain field produced by an impurity and the capability of this impurity to trap (Fig. 4). The escape appears not to be correlated at all. The values of the volume expansion induced by these impurities have been given in Table $\mathrm{V}$ for comparison.

Neutron diffraction experiments on Al doped with the same impurities have shown that shortrange displacements of the host atoms exist also in the case of small or negligible long-range dilatations. ${ }^{61}$ We conclude that the depths and radii of these trapping regions depend on the detailed electronic structure around each type of impurity and cannot be predicted from the long-range behavior.

It is also impossible at present to extract direct information from the position of the maxima of $\sigma(T)$, since they are determined by an interplay of capture and release processes which can be quite complicated for structured traps. The rise of $\sigma(T)$ to the trapping peaks shows secondary maxima for $\mathrm{Li}$ and $\mathrm{Mg}$ that may be caused by trapping at an intermediate shallow level, i.e., by the internal structure of the trap. The deviations from linear $T$ dependence found for these samples may be due to the fact that such effects have not been taken into account.

\section{B. Region below $1 \mathrm{~K}$}

From the analysis of muon depolarization in pure $\mathrm{Al}$ and the Al alloys the following facts have emerged.

The apparent correlation time behaves approximately as (i) $\tau_{c} \propto c^{0.76}$ and (ii) $\tau_{c} \propto T^{-0.6}$.

(iii) Impurities with small (Li) or negligible (Ag) volume dilatations give rise to the same depolarization (apart from a small quantitative factor) as the impurity with large volume dilatation (Mn).

(iv) The muons occupy $O$ sites at the lowest temperatures in contrast to the $T$ sites found at high temperature $(15 \mathrm{~K})$ for the same $\mathrm{Mn}$-doped samples.

(v) The muon is localized at or near one single

TABLE V. Lattice expansion of Al due to substitutional impurities, deduced from Pearson (Ref. 24).

\begin{tabular}{ccccc}
\hline \hline & & & Impurity & \\
& $\mathrm{Mn}$ & $\mathrm{Mg}$ & $\mathrm{Li}$ & $\mathrm{Ag}$ \\
\hline$\frac{\Delta a}{a}$ for 1 at. $\%$ & $-1.5 \times 10^{-3}$ & $0.99 \times 10^{-3}$ & $-1.1 \times 10^{-4}$ & $<10^{-5}$ \\
$\Delta V\left(\AA^{3}\right)$ & -7.4 & 4.9 & -0.54 & $<0.05$ \\
\hline \hline
\end{tabular}


interstitial site, for most of its lifetime, as deduced from the electric field gradient, at least in $57 \mathrm{ppm}$ $A l \mathrm{Mn}$ at the lowest temperatures.

We cannot offer a unique and simple explanation of all facts noted above. Instead we will discuss different possible explanations and try to extract the most likely one. We will first examine whether the observed facts can be understood from motional processes in the bulk material which do not change their character during the lifetime of the muons, i.e., we disregard trapping.

We first consider uniform incoherent motion of the muons down to the lowest temperatures. The rise of $\sigma(T)$ with $T \rightarrow 0$ would then be caused by the gradual freezing of this process. A candidate is the apparent one-phonon process whose existence was supported by the $T>1 \mathrm{~K}$ data. The $T$ dependence is in qualitative, but not quantitative, agreement with such a model which would predict $\tau_{c}^{-1} \propto T$ (for $|\Delta E|<<k_{B} T$ ). The strain-induced part of this mechanism would, however, produce an increased jump rate (decrease of $\sigma$ ) with increasing $c$, contrary to the reversed dependence on $c$ [see (i) above]. The rate for jumps between $O$ and $T$ sites would be independent of $c$ at these low concentrations. Both possibilities can therefore be discarded. Since the motion process should take place mainly in regions relatively far from the impurities where Eq. (9) should be valid, it would also be difficult to explain the independence on the kind of impurity (iii) in this context.

Secondly we discuss whether the rise of $\sigma(T)$ as $T \rightarrow 0$ might be due to uniform coherent motion. According to the discussion of polarization decay in the case of coherent diffusion in Sec. III F, the correlation time should be inversely proportional to $D_{\text {coh }}$, cf. Eq. (27). The result for $\tau_{c}$ would then imply $D_{\text {coh }} \propto c^{-0.76} T^{0.6}$. Such behavior of the diffusion coefficient would be in qualitative agreement with the scattering on impurities as the dominating scattering mechanism. This would require that all other scattering mechanisms could be neglected in comparison to impurity scattering. We believe such an explanation to be unlikely. First, rough quantitative estimates of the scattering rate on impurities indicate that this rate is much smaller than required to explain the data for $\tau_{c}$. Second, the analysis of the data above $1 \mathrm{~K}$ strongly suggests that incoherent hopping of localized muons occurs in that temperature range. It is difficult to imagine that somewhat below $1 \mathrm{~K}$ the muons then abruptly propagate freely, scattering only on the impurities. Third, the data on the electric field gradient (v) in $57 \mathrm{ppm} A l \mathrm{Mn}$ at 40
$\mathrm{mK}$ show that the muon is localized at or near a single interstitial site. This is also in contradiction to the assumption of free coherent motion between the impurities, since the muon would be delocalized in that case.

Since a simple uniform diffusion cannot explain the data observed in the region below $1 \mathrm{~K}$ we have to consider more complicated models involving a change of the motional process during the muon lifetime.

The least complicated of these is again trapping preceded by a diffusive motion. At the very lowest temperatures the muons are assumed to stay in the traps over their remaining lifetime.

The fit of the data to the two-state model resulted in

$$
\tau_{1}^{-1} \propto c^{0.76} T^{-0.6}
$$

This interpretation of the damping rate presupposes that strong motional narrowing prevails in the diffusive state.

The inverse dependence on temperature below 1 $\mathrm{K}$ immediately excludes any phonon-induced process for reaching such low-temperature traps. The low- $T$ traps must therefore be reached by a coherent process. Since the conditions are somewhat different if the transport is capture controlled or diffusion controlled the two cases will now be discussed separately.

The capture-controlled process requires a coherence length $l \gg r_{t}$. As discussed above, this situation is not likely to occur because of scattering processes due to electrons and remaining impurities. Still, if it were operative, $\tau_{1}^{-1}=\sigma_{\mathrm{abs}} v c$ should be independent of $T$ for deep traps [see Eq. (20) and the subsequent text], which is not observed. More complicated $T$ dependencies than $\propto v^{-1}$ for $\sigma_{\text {abs }}$ could occur for extended traps but are expected to be typical for each impurity whereas we observe an almost universal behavior.

The most appealing of the "simple" trapping processes is therefore a diffusion-controlled trapping preceded by a coherent motion of the type $l<r_{t}$. In this case the capture rate is expected to be given by Eq. (19) with $D \propto v^{2} \tau$. With $z J_{\text {eff }}$ estimated to be of the order of $1 \mathrm{meV}$ we expect $z J_{\text {eff }} \gg k_{B} T$ to hold below $1 \mathrm{~K}$ and the velocity of a thermalized muon should be proportional to $T^{1 / 2}$. We deduce from this interpretation the behavior of the transport scattering rate which delimits coherent diffusion; explicitly $\tau^{-1} \propto c^{0.24} T^{1.6}$. The dependencies of this scattering rate have been listed in Table I for various possible processes. 
We note that our result for $\tau^{-1}$ is in obvious disagreement with the behavior resulting from phonon scattering. In other words, the $T^{9}$ behavior predicted by Kagan and Klinger, ${ }^{25}$ or variants of it, are not observed. The behavior of the transport scattering rate given above agrees roughly with that resulting from electron scattering. However, the residual dependence on concentration, and the precise temperature power are not understood.

The fact that trapping occurs in $O$ sites instead of $T$ sites as at high temperature is a serious open problem; it is probably connected with the different nature of the process by which the traps are reached (the low- $T$ trapping might occur further out from the impurity centers). It could be noted that the smaller depolarization for Li doping compared to Mn doping of similar concentration (70 ppm) means a longer trapping time, i.e., time of stay in the coherent regime for the case of Li doping. Although the difference is not large, this fact indicates a smaller trapping radius for $\mathrm{Li}$ than for Mn.

All the observed facts [(i) - (v) above] can therefore be said to fit qualitatively into a picture of coherent motion before diffusion-limited trapping. The precise power laws are still not reproduced, but the data provide strong evidence for the existence of coherent motion in this temperature range, for samples of sufficient purity.

One can also consider more complicated physical pictures, but we have not yet worked out these in detail. For instance, one can assume that the muons first perform rapid coherent motion and then enter into regions where they get localized but continue to diffuse by an incoherent process. The region where the transition between both types of motion occurs should be strongly disturbed (of the order of $z J_{\text {eff }}$ ); on the other hand, only energies of the order of $k_{B} T$ are available for thermal activation. Hence the incoherent process would most likely consist of a sequence of steps which lead more deeply into the trap. This would give a temperature-independent contribution to the depolarization, and the temperature variation deduced for coherent diffusion would be slightly increased.

The interpretation of our results below and above $1 \mathrm{~K}$ implies a transition from coherent to incoherent diffusion around this temperature. By comparing the experimental transition temperature of $\sim 1 \mathrm{~K}$ with the prediction of small-polaron theory, which is of the order of some tenths of the Debye temperature, again large discrepancies between experiment and theory become obvious. A possible way to overcome these difficulties might be the consideration of electron-scattering processes that are not related to the vibrational properties of the Al lattice.

Finally, some comments should be made on the possibility of capture from a metastable, non-selftrapped state, as suggested by Browne and Stoneham. ${ }^{11}$ In this theory the muons perform diffusive motion in extended states with a mean free path limited by scattering on the impurities. The authors assume $l \propto c^{-1 / 3}$ in apparent contradiction to the accepted theory ${ }^{62}$ of transport of, e.g., electrons in band states. If transport is limited by scattering on impurities, $l$ should be inversely proportional to c. Furthermore, they use the expression (19) for diffusion-limited trapping, whereas their assumption on the metastable state $\left(l>r_{t}\right)$ means a capture-limited trapping. Although a motion in a metastable state before trapping at low $T$ cannot be excluded, it is doubtful whether their theory can be modified to give a better description of the results for $\mathrm{Al}$ and $\mathrm{Al}$ alloys than the one presented here.

\section{CONCLUSIONS}

A number of questions concerning the nature of motion of light interstitials, especially for the case of positive muons, were raised in the introduction and in the review of the theory of diffusion. Summing up the arguments of the preceding section, we can draw the following conclusions from the present experiments:

(1) The diffusion mechanisms of muons in aluminum appear to be different above and below $1 \mathrm{~K}$. Above $1 \mathrm{~K}$ the muons perform incoherent hopping, whereas below $1 \mathrm{~K}$ they diffuse by coherent transfer at least in parts of the sample if the samples are sufficiently pure.

(2) Above $1 \mathrm{~K}$, muons are trapped at substitutional impurities. The capture process is diffusion-limited trapping at single impurities.

(3) The linear temperature dependence found for the incoherent hopping rate is in contradiction to conventional small-polaron theory; it is typical for a one-phonon process. However, the theories of one-phonon processes do not apply to the present case, with the exception of possible alternating transitions between $O$ and $T$ sites.

(4) The trapping regions around each impurity are extended, comprising many interstitial sites. The size of these regions is not directly correlated to the long-range strain field of the impurity used.

(5) In pure aluminum the motional process is of such nature that practically complete motional nar- 
rowing takes place down to $100 \mathrm{mK}$. Because of motional narrowing, the regime of coherent motion can only be studied indirectly in $\mu$ SR experiments as a stage preceding trapping at low temperatures.

(6) The analysis of depolarization below $1 \mathrm{~K}$ in aluminum alloys suggests trapping controlled by coherent diffusion. The resulting diffusion coefficient is then proportional to $T^{-0.6}$. The temperature dependence is in disagreement with the predictions of conventional small-polaron theory, but it is in rough agreement with the predictions of scattering of conduction electrons on the muon.

(7) The transition temperature of approximately $1 \mathrm{~K}$ between the region of incoherent hopping and the region of coherent transfer is lower than that predicted by small-polaron theory.

(8) The $O$ sites are the lowest-energy sites for muons at the lowest temperatures. At intermediate temperatures the muons seem to move over $T$ sites as well as $O$ sites. At the $\mathrm{Mn}$-trapping peak in $\mathrm{Al}$ the $T$ site is the most stable one, probably lowered in energy by the closeness of an Mn atom at a substitutional site.

In our opinion the essential results of the present investigation are (i) clear evidence for both incoherent hopping and coherent motion of muons in pure aluminum, and (ii) the fact that the results are not adequately described by the current smallpolaron theory of the motion of light interstitials in metals, which predicts strong temperature dependencies of incoherent hopping and coherent diffusion. The weak temperature dependence of the coefficient of coherent diffusion, as well as the low transition temperature between dominating coherent diffusion and dominant incoherent hopping, indicate that electron scattering may play an important role at low temperatures. Since aluminum is the only metal which has been studied so far systematically at low temperature, it is of great importance to extend the investigations to other materials in order to find out whether the observed diffusion phenomena are of a universal nature.

Note added in proof. Some points that we criticized in the preliminary version of Ref. 11, especially the assumption $1 \propto c^{-1 / 3}$, have been corrected in the published version. However, our final conclusion about the applicability of this mechanism is not altered.

\section{ACKNOWLEDGMENTS}

We are indebted to G. Jungerberg for technical assistance in preparing the samples and to $H$. Beske and M. W. Brunner for analytical support. The help of J. Chappert and A. Kuijk in some of the experiments is gratefully acknowledged. We thank J. W. Haus, J. Jäckle, and K. Kitahara for discussions on the theory of muon diffusion.
${ }^{1}$ I. I. Gurevich, E. A. Meleshko, I. A. Muratowa, B. A. Nikolsky, V. S. Roganov, V. I. Selivanov, and B. V. Sokolov, Phys. Lett. 40A, 143 (1972).

2Proceedings of the First International Topical Meeting on Muon Spin Rotation, Rorschach, 1978, edited by F. N. Gygax, W. Kündig, and P. F. Meier [Hyperfine Interact. $\underline{6},(1979)]$.

${ }^{3}$ Proceedings of the Second International Topical Meeting on Muon Spin Rotation, Vancouver, 1980, edited by J. H. Brewer and P. W. Percival [Hyperfine Interact. $\underline{8},(1981)]$.

${ }^{4}$ O. Hartmann, E. Karlsson, P. Pernestål, M. Borghini, T. O. Niinikoski, and L. O. Norlin, Phys. Lett. $\underline{61 \mathrm{~A}}$, 141 (1977).

${ }^{5}$ O. Hartmann, E. Karlsson, L. O. Norlin, D. Richter, and T. O. Niinikoski, Phys. Rev. Lett. 41, 1055 (1978).

${ }^{6}$ W. J. Kossler, A. T. Fiory, W. F. Lankford, K. G. Lynn, R. P. Minnich, and C. E. Stronach, Hyperfine Interact. 6, 295 (1979).

${ }^{7}$ O. Hartmann, E. Karlsson, L. O. Norlin, T. O. Niinikoski, K. W. Kehr, D. Richter, J. M. Welter, A. Yaonanc, and L. LeHéricy, Phys. Rev. Lett. 44, 337 (1980).
${ }^{8}$ J. H. Brewer, K. M. Crowe, F. N. Gygax, and A. Schenck, in Muon Physics, Vol. III: Chemistry and Solids, edited by V. W. Hughes and C. S. Wu (Academic, New York, 1975), p. 3.

${ }^{9}$ D. Emin, Adv. Phys. 22, 57 (1973).

${ }^{10}$ D. Emin and T. Holstein, Phys. Rev. Lett. 36,323 (1976).

${ }^{11}$ A. M. Browne and A. M. Stoneham, J. Phys. C $\underline{15}$, 2709 (1982).

${ }^{12}$ M. Manninen and R. M. Nieminen, J. Phys. F 9, 1333 (1979).

${ }^{13}$ L. M. Kahn, F. Perrot, and M. Rasolt, Phys. Rev. B 21, 5594 (1980).

${ }^{14}$ F. Perrot, M. Rasolt, Phys. Rev. B 23, 6534 (1981).

${ }^{15}$ C. Berthier, M. Monier, J. Phys. F 7 , 515 (1977).

16J. P. Bugeat, A. C. Chami, and E. Ligeon, Phys. Lett. 58A, 127 (1976).

17 J. P. Bugeat and E. Ligeon, Phys. Lett, 71A, 93 (1979).

${ }^{18}$ T. Holstein, Ann. Phys. (N.Y.) $\underline{8}, 325$ (1959); $\underline{8}, 343$ (1959).

${ }^{19}$ A. M. Stoneham, J. Phys. F $\underline{2}, 417$ (1972).

${ }^{20}$ P. W. Anderson, Phys. Rev. 109, 1492 (1958).

${ }^{21}$ See, e.g., D. Thouless, in Ill-Condensed Matter, Les 
Houches Session XXXI, 1978, edited by R. Balian, R. Maynard, and G. Toulouse (North-Holland, Amsterdam, 1979), p. 1.

${ }^{22}$ G. Leibfried and N. Breuer, Point Defects in Metals I, Vol. 81 of Springer Tracts on Modern Physics, edited by G. Höhler (Springer, Berlin, 1978), p. 163.

${ }^{23}$ B. Baranowski, S. Majchrzak, and T. B. Flanagan, J. Phys. F 1 , 258 (1971).

${ }^{24}$ W. B. Pearson, Lattice Spacings and Structure of Metals and Alloys (Pergamon, Oxford, 1958), p. 346.

${ }^{25}$ Y. Kagan and M. J. Klinger, J. Phys. C I, 2791 (1974).

${ }^{26} \mathrm{~K}$. W. Kehr, in Proceedings of the Second Japan Institute of Metals International Symposium on Hydrogen on Metals, Minakami, 1979 [Trans. Jpn. Inst. Met. Suppl. 21, 181 (1980)].

${ }^{27}$ A. F. Andreev, and I. M. Lifshitz, Zh. Eksp. Teor. Fiz. 56, 2057 (1969) [Sov. Phys.-JETP 29, 1107 (1969)].

${ }^{28}$ J. Jäckle and K. W. Kehr (unpublished).

${ }^{29}$ A. F. Ioffe, A. R. Regel, Prog. Semicond. 4, 237 (1960).

${ }^{30}$ C. P. Flynn and A. M. Stoneham, Phys. Rev. B 1 , 3966 (1970).

${ }^{31}$ S. Fujii, J. Phys. Soc. Jpn. 46, 1833 (1979).

${ }^{32}$ H. Teichler, Phys. Lett. 64A, 78 (1977).

${ }^{33}$ M. Camani, F. N. Gygax, W. Rüegg, A. Schenck, and H. Schilling, Phys. Rev. Lett. 푸, 836 (1977).

${ }^{34} \mathrm{D}$. Herlach, in Recent Developments in Condensed Matter Physics, edited by J. T. Devreese (Plenum, New York, 1981), Vol. 1, p. 93.

35J. A. Sussmann, Phys. Kondens. Mater. 2, 146 (1964).

${ }^{36}$ H. Teichler and A. Seeger, Phys. Lett. 82A, 91 (1981).

${ }^{37}$ A. Yaouanc, Phys. Lett. 87A, 423 (1982).

${ }^{38}$ K. G. Petzinger (unpublished).

${ }^{39}$ M. Borghini, T. O. Niinikoski, J. C. Soulié, O. Hartmann, E. Karlsson, L. O. Norlin, K. Pernestål, K. W. Kehr, D. Richter, and E. Walker, Phys. Rev. Lett. 40, 1723 (1978).

${ }^{40}$ E. Karlsson, Hyperfine Interact. $\underline{8}, 647$ (1981).

${ }^{41}$ D. Richter, in Nuclear and Electron Resonance Spectroscopies Applied to Materials Science, Proceedings of the Materials Research Society's Annual Meeting, edited by E. N. Kaufmann and G. K. Shenoy
(North-Holland, New York, 1981), p. 233.

${ }^{42}$ One could imagine traps which consist of high barriers, and where the ground-state level is not lowered compared to the host. The capture of a muon on such a trap would be extremely unlikely.

${ }^{43}$ T. R. Waite, Phys. Rev. 107, 463 (1957).

${ }^{44}$ K. Schröder and K. Dettmann, Z. Phys. B $\underline{22}, 343$ (1975).

${ }^{45}$ C. H. Hodges, J. Phys. F 4, L230 (1974).

46I. K. Mackenzie, T. L. Khoo, A. B. McDonald, and B. T. A. McKee, Phys. Rev. Lett. 19, 946 (1967).

${ }^{47}$ R. Lenzi, P. Podini, R. Reverberi, and K. Pernestål, Nucl. Instrum. Methods 150, 575 (1978).

${ }^{48}$ K. W. Kehr, D. Richter, J. M. Welter, O. Hartmann, L. O. Norlin, E. Karlsson, T. O. Niinikoski, J. Chappert, and A. Yaouanc, Hyperfine Interact. $\underline{8}, 681$ (1981).

${ }^{49}$ W. J. Kossler, A. T. Fiory, W. F. Lankford, L. Lindemuth, K. G. Lynn, S. Majahan, R. P. Minnich, K. G. Petzinger, and C. E. Stronach, Phys. Rev. Lett. 41, 1558 (1978).

${ }^{50} \mathrm{~A}$. Schenck, in Nuclear and Particle Physics at Intermediate Energies, edited by J. B. Warren (Plenum, New York, 1976), p. 159.

${ }^{51}$ K. W. Kehr, G. Honig, and D. Richter, Z. Phys. B 32, 49 (1978).

${ }^{52}$ K. W. Kehr, D. Richter, and G. Honig, Hyperfine Interact. $\underline{6}, 219$ (1979).

${ }^{53}$ K. G. Petzinger, R. L. Munjal, and E. Zaremba, Hyperfine Interact. $\underline{6}, 223$ (1979).

${ }^{54} \mathrm{R}$. Kubo, in Fluctuation, Relaxation, and Resonance in Magnetic Systems, edited by D. Ter Haar (Oliver and Boyd, Edinburgh, 1962), p. 23.

${ }^{55}$ R. Kubo, J. Phys. Soc. Jpn. 9, 935 (1954).

${ }^{56} \mathrm{O}$. Hartmann (unpublished).

${ }^{57}$ T. McMullen and E. Zaremba, Phys. Rev. B 18, 3026 (1978).

58J. A. Van Vleck, Phys. Rev. 74, 1168 (1948).

${ }^{59}$ O. Hartmann, Phys. Rev. Lett. $\underline{39}, 832$ (1977).

${ }^{60}$ D. Richter and T. Springer, Phys. Rev. B $\underline{18}, 126$ (1978).

${ }^{61} \mathrm{~K}$. Werner, private communication.

62J. M. Ziman, Electrons and Phonons (Clarendon, Oxford, 1980). 